\title{
Galaxies with Wolf-Rayet signatures in the low-redshift Universe
}

\section{A survey using the Sloan Digital Sky Survey ${ }^{\star}$}

\author{
J. Brinchmann ${ }^{1,2}$, D. Kunth ${ }^{3}$, and F. Durret ${ }^{3}$ \\ ${ }^{1}$ Leiden Observatory, Leiden University, PO Box 9513, 2300 RA Leiden, The Netherlands \\ e-mail: jarle@strw.leidenuniv.nl \\ 2 Centro de Astrofísica da Universidade do Porto, Rua das Estrelas S/N, 4150-762 Porto, Portugal \\ 3 Institut d'Astrophysique de Paris, UMR 7095 CNRS, Université Pierre \& Marie Curie, 98 bis boulevard Arago, 75014 Paris, France
}

Received 14 March 2008 / Accepted 10 April 2008

\begin{abstract}
Context. The availability of large spectroscopic datasets has opened up the possibility of constructing large samples of rare objects in a systematic manner.

Aims. The goal of this study is to analyse the properties of galaxies showing Wolf-Rayet features in their optical spectrum using spectra from the Sloan Digital Sky Survey Release 6. With this unprecedentedly large sample we aim to constrain the properties of the Wolf-Rayet phase and its impact on the surrounding interstellar medium.

Methods. We carried out very careful continuum subtraction on all galaxies with equivalent widths of $\mathrm{H} \beta>2 \AA$ in emission and identify Wolf-Rayet features using a mixture of automatic and visual classification. We combined this with spectroscopic and photometric information from the SDSS and derive metal abundances using a number of methods.

Results. We find a total of 570 galaxies with significant Wolf-Rayet (WR) features and a further 1115 potential candidates, several times more than even the largest heterogeneously assembled catalogues. We discuss in detail the properties of galaxies showing WolfRayet features with a focus on their empirical properties. We are able to accurately quantify the incidence of Wolf-Rayet galaxies with metal abundance and show that the likelihood of otherwise similar galaxies showing Wolf-Rayet features increases with increasing metallicity, but that WR features are found in galaxies of a wide range in morphology. The large sample allows us to show explicitly that there are systematic differences in the metal abundances of WR and non-WR galaxies. The most striking result is that, below $E W(\mathrm{H} \beta)=100 \AA$, Wolf-Rayet galaxies show an elevated N/O relative to non-WR galaxies. We interpret this as a rapid enrichment of the ISM from WR winds. We also show that the model predictions for WR features strongly disagree with the observations at low metallicity; while they do agree quite well with the data at solar abundances. We discuss possible reasons for this and show that models incorporating binary evolution reproduce the low-metallicity results reasonably well. Finally we combine the WR sample with a sample of galaxies with nebular $\mathrm{He}$ II $\lambda 4686$ to show that, at $12+\log \mathrm{O} / \mathrm{H}<8$, the main sources of He II ionising photons appears to be $\mathrm{O}$ stars, arguing for a less dense stellar wind at these metallicities, while at higher abundances WN stars might increasingly dominate the ionisation budget.
\end{abstract}

Key words. stars: Wolf-Rayet - galaxies: abundances - galaxies: evolution - galaxies: starburst - galaxies: fundamental parameters

\section{Introduction}

The presence of features originating from Wolf-Rayet stars in the spectra of galaxies provide considerable information on the recent star formation activity in these systems and can be used to study the properties of the Wolf-Rayet stars and place strong constraints on the massive end of the initial mass function (IMF). Wolf-Rayet features in galaxy spectra are interesting because the first Wolf-Rayet stars typically start to appear about $2 \times$ $10^{6}$ years after a star formation episode and disappear within some $5 \times 10^{6}$ years. Thus they provide a high-resolution temporal tracer of the recent star formation activity of a galaxy.

Galaxies containing the signatures of Wolf-Rayet stars have been known for several decades. Beginning with the first detection of Wolf-Rayet features in the galaxy $\mathrm{He} 2-10$ by

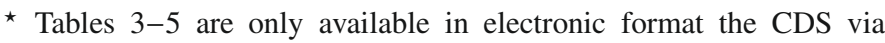
anonymous ftp to cdsarc.u-strasbg.fr (130.79.128.5) or via http://cdsweb.u-strasbg.fr/cgi-bin/qcat?J/A+A/485/657
Allen et al. (1976), a number of these galaxies have been reported, some in systematic searches (e.g. Kunth \& Joubert 1985), but mostly serendipitously. In fact the first early reports of the detection of Wolf-Rayet stellar features in galaxy spectra were incidental results from studies of starbursting low-metallicity galaxies, typically blue compact dwarfs, aiming at obtaining spectra of high $S / N$ for deriving the primordial He abundance (Kunth \& Sargent 1983). Kunth et al. (1981) realised from their first spectra of NGC 3125 that the presence of Wolf-Rayet stars provides a powerful constraint on the recent star formation in a galaxy. Soon after these discoveries, the term "WR galaxy" was introduced by Osterbrock \& Cohen (1982) and Conti (1991). This term should be used with caution, however, since depending on the distance of the object and the spatial extension of the observation it may refer to for instance "extragalactic HII regions" that are typical of the outskirts of spiral galaxies or quite frequently to the nucleus of a powerful starburst. The properties and importance of these phenomena in both 
cases are completely different (in terms of metallicity, luminosity, number of WR stars, etc.). As a consequence the WR galaxy definition hides a loose concept. WR galaxies are found among a very wide variety of morphological types ranging from lowmass, blue compact low-metallicity dwarf galaxies to massive spirals, luminous mergers, IRAS galaxies and even LINERS or Seyfert 2 (Ho et al. 1995; Heckman \& Leitherer 1997; Contini et al. 2001).

The possibility that WR stars are seen in central cluster galaxies has been reported by Allen (1995). The use of WolfRayet galaxies to constrain the recent star formation should be seen in the context of other techniques and indeed in the context of the other properties of galaxies. Despite these drawbacks we shall refer to the WR galaxy nomenclature for simplicity. Since the compilation of Conti (1991) that included 37 objects, the most recent catalogues are from Schaerer et al. (1999b) and Guseva et al. (2000) with more than 130 objects and the study using SDSS by Zhang et al. (2007) which includes 174 objects and is currently the largest survey for WR galaxies, although as we will see only 101 of these satisfy the criteria to be included into our sample.

Observationally most, if not all, WR galaxies are identified whenever their integrated spectra show a broad He II $\lambda 4686 \AA$ emission feature that is thought to originate in the stellar winds of these WR stars. Additional features are also found, for instance N III $\lambda 4640 \AA$ and/or C III/IV $\lambda 4650 \AA$ as well as C IV $\lambda 5808 \AA$ that was shown to be present in many cases (Schaerer et al. 1999a) and originate from WN and WC stars. Most recent studies have attempted to reproduce the number of WR stars responsible for the observed stellar emission features. The model predictions for this are strongly dependent on metallicity as stellar winds show a strong metallicity dependence (e.g. Nugis \& Lamers 2000). Simple calculations involving the $\mathrm{H} \beta$ emission line combined with the strength of the so-called WR bump at $\lambda 4686 \AA$ already give a hint (Kunth \& Sargent 1981), but more refined theoretical evolutionary models predict that at fixed metallicity the WR/O ratio strongly varies with the age of the starburst (Mas-Hesse \& Kunth 1991; Maeder \& Meynet 1994; Schaerer \& Vacca 1998). It is found that this ratio reaches a maximum of 1 for solar metallicity but decreases to 0.02 when the metallicity decreases to $Z_{\odot} / 50$. This latter point remains a concern in the sense that it does not quite reproduce the observational fact that some low metallicity starburst galaxies such as I Zw 18 contain a WR population that largely outnumbers model predictions (Legrand et al. 1997; de Mello et al. 1998), forcing one to envisage other channels for the WR phase (such as the binary channel hypothesis), revise the models or question the adopted luminosity of the WCE stars in metal-poor models (Fernandes et al. 2004). On the high metallicity side, Pindao et al. (2002) in contrast find too low a $I$ (WRbump) $/ I(\mathrm{H} \beta)$ ratio compared with model predictions, suggesting again that WR luminosities are not correctly calculated. The model predictions have recently been contrasted with observed broad WR emission features by Fernandes et al. (2004) and the application of existing models to a sample of WR galaxies from the SDSS was shown to imply significant modifications of the IMF by Zhang et al. (2007), which might equally be taken to indicate significant problems with the current sample of models. We return to this issue in Sect. 10.

While interesting in their own rights most of the galaxies under investigation here show very strong star formation activity with many showing $S F R / M_{*}$ similar to that of high redshift Lyman-break galaxies (e.g. Steidel et al. 2001, 2003;
Shapley et al. 2003). Several of these galaxies are found to show potential Wolf-Rayet spectral features, in particular the He II $\lambda 1640 \AA$ feature (e.g. Lowenthal et al. 1991, 1997; Kobulnicky \& Koo 2000; Shapley et al. 2003), hence the present compilation might be suitable as a local comparison sample.

In addition there is mounting evidence that the progenitors of long gamma-ray burst (GRB) events are related to the WolfRayet phase (e.g. Woosley \& Bloom 2006) and Hammer et al. (2006) were able to show that nearby gamma-ray burst host galaxies often show Wolf-Rayet stars, although these do not necessarily coincide with the location of the gamma-ray bursts. Since studies of Wolf-Rayet galaxies allow one to place constraints on the models for massive star evolution they may also provide insight into the properties of GRB progenitors. However to achieve this it is essential that the sample of Wolf-Rayet galaxies has a well characterised selection function and covers a wide range in metallicity and star-burst age. In previous studies the emphasis has often been on heterogeneous samples, and even when the sample selection is clearly stated, the samples have been selected to contain only systems with high equivalent width Balmer lines. Metal-rich galaxies are known to be on average more massive (Tremonti et al. 2004) so this would bias against metal rich galaxies.

In this paper we carry out a survey for Wolf-Rayet features in galaxies covering a very wide range in properties using the Sloan Digital Sky Survey Data Release 6 (SDSS DR6, Adelman-McCarthy et al. 2008). We introduce the data we use in Sect. 2 and discuss our pre-selection of candidates with WolfRayet emission features in Sect. 3. Our method to measure WolfRayet features is outlined in Sect. 4 where we verify that the distribution of line widths is similar to that seen in Galactic and Magellanic WR stars. Section 5 describes the overall properties of the sample and place it in the context of the full SDSS. The abundance of Wolf-Rayet galaxies with redshift is discussed in Sect. 6 and their emission line properties in Sect. 7. We discuss evidence of large-scale pollution of the host galaxy ISM by WR winds in Fig. 9. The modelling of WR features in galaxies is discussed in Sect. 10. We conclude in Sect. 11.

\section{Data}

We base our search for Wolf-Rayet galaxies on the Sloan Digital Sky Survey (SDSS, York et al. 2000). The SDSS uses a dedicated $2.5 \mathrm{~m}$ telescope (Gunn et al. 2006) and obtains five-band images using a drift-scan technique (Gunn et al. 1998) and spectra using a double-barred fibre spectrograph with $3^{\prime \prime}$ fibre apertures and a total of 640 fibres per plate. The tiling algorithm used is discussed in Blanton et al. (2003), the photometric system by Fukugita et al. (1996) and the photometric calibration is discussed in Smith et al. (2002a) and Tucker et al. (2006). The photometric stability is monitored using a dedicated set-up discussed by Hogg et al. (2001) and objects are detected using the Photo pipeline discussed in Lupton (2008). The astrometric precision is better than 0.1 arcsec for the objects considered here (Pier et al. 2003).

We take as starting point the SDSS Data Release 6 (Adelman-McCarthy et al. 2008) which contains a total of 1271680 spectra. Our analysis method is optimised for normal galaxy spectra so we limit our sample to spectra that are classified by the SDSS pipeline to be a galaxy spectrum (spectrotype="galaxy"). This excludes all QSOs and essentially all type 1 Seyferts. Note also that we do include duplicate observations, a total of 91825 , and will use these below. These initial cuts reduce our sample to 796912 spectra. 
We also require that the equivalent width of $\mathrm{H} \beta$ in emission is $>2 \AA$. This leads to a total of $N=307210$ spectra. We do not impose a signal-to-noise $(S / N)$ cut, but $96 \%$ of these spectra have $\mathrm{H} \beta$ detected at $S / N>3$. For the analysis of the ionisation properties of the galaxies we need to apply a $S / N$ cut and define a sub-sample where the spectra have $S / N>3$ in $\mathrm{H} \beta$, [O III] 5007 , $\mathrm{H} \alpha$ and [N II]6584, after the uncertainty estimates are adjusted as discussed in Sect. 2.1. This sub-sample contains 224939 spectra.

The minimum requirement of $E W(\mathrm{H} \beta)>2 \AA$, ensures that almost all spectra under consideration have significant emission lines present and/or a weak continuum. One might worry that this could lead us to miss a significant number of spectra with Wolf-Rayet features. By extrapolating the trend of number of spectra with WR features versus $E W(\mathrm{H} \beta)$ shown in Fig. 7 below we estimate that less than $10(0.002 \%)$ spectra with Wolf-Rayet features are excluded by this cut.

While the SDSS database is a unique resource, it is well worth pointing out that it is not optimally suited for the study of low redshift galaxies. This is in part because the low wavelength cut-off of the spectrograph is $\sim 3800 \AA$, which means that the important [O II] 3727 line falls outside the spectral range for $z<0.02$. As pointed out by Kniazev et al. (2004) this can to some extent be remedied by using the [O II] 7320, 7330 quadruplet instead, but this is a much weaker line. Furthermore, the spectroscopic target selection is done in the $r$-band and while this ensures a fairly good sampling of the galaxy population based on their stellar content, it will include fewer strongly star-forming systems, such as the galaxies showing Wolf-Rayet features discussed here, than, for example, a $B$-band selection.

Finally, the SDSS spectroscopic target selection is done based on an analysis of the SDSS images using the Photo pipeline, which detects objects using a sophisticated segmentation routine. This works very well in general, but for the strongly star forming systems we are interested in here, the galaxies might be split into several separate objects by the segmentation process.

A careful re-analysis of the images is beyond the scope of this paper but is discussed by Blanton et al. (2005). What concerns us is that the spectra frequently target the brightest $\mathrm{H}$ II regions in nearby galaxies, often significantly displaced from the galaxy centre and we will return to this issue below.

The spectra of all target galaxies have been re-analysed using the two-step procedure outlined in Appendix A. This is based on the pipeline discussed by Tremonti et al. (2004, hereafter T04) and the resulting fits are then refined to provide flux and equivalent width measurements for 40 emission lines, as well as continuum indices and other derived parameters.

\subsection{Empirical adjustment of uncertainty estimates}

The SDSS pipeline provides an uncertainty estimate for each pixel in a spectrum including internal error sources such as Poissonian noise from the sky and detectors. These uncertainty estimates do not, however, include external error sources such as uncertainties in the overall flux calibration and in the continuum subtraction. To accurately check and adjust the uncertainty estimates one needs duplicate observations of galaxies.

The SDSS targets some galaxies twice to allow for systematic checks of this kind, as first exploited by Gómez et al. (2003). The SDSS DR6 contains 86156 duplicate observations of galaxies, and we use these to adjust the standard uncertainty estimates.
To compare two spectra of the same galaxy we calculate:

$$
\Delta_{N}=\frac{f^{\text {first }}-f^{\text {second }}}{\sqrt{\sigma_{\text {first }}^{2}+\sigma_{\text {second }}^{2}}}
$$

where $f$ stands for the line flux and $\sigma$ represents the associated formal uncertainty. Assuming Gaussian noise, $\Delta_{N}$ should be a normal random variable with a standard deviation of 1 if the error estimates are accurate. We use the difference between the observed spread and that expected to determine correction factors to the uncertainty estimates. The typical corrections to the uncertainty estimates are $20-50 \%$ for forbidden lines and a factor of 2-2.5 for Balmer lines and for [O II] 3727.

\subsection{Internal dust attenuation}

The spectra are corrected for Galactic attenuation using the Schlegel et al. (1998) dust maps before running the fitting pipeline. To adjust for internal attenuation we use the standard approach of comparing the ratio $\mathrm{H} \alpha / \mathrm{H} \beta$ to the expected Case B value. We use the Case $B$ value appropriate for the value of $T_{\mathrm{e}}$ estimated as discussed in the following section. We assume a simple, $\tau \propto \lambda^{-1.3}$, dust law as advocated by Charlot \& Fall (2000). The details of the dust correction do not influence the results below, nearly identical results would be obtained if a fixed $\mathrm{H} \alpha / \mathrm{H} \beta$ ratio was assumed.

While this recipe for dust correction is sufficient for nebular emission lines, it is not entirely clear whether it is appropriate to assume the same dust attenuation for the Wolf-Rayet features as for the nebular lines. In the following we will assume that the attenuation at $\mathrm{H} \beta$ and the blue bump is the same. This is likely to give an upper limit to the attenuation of the Wolf-Rayet bump.

\subsection{Abundance measurements}

Since our sample spans a wider range in galaxy properties than is normally encountered in Wolf-Rayet galaxy studies it is more complicated to estimate element abundances in a uniform way for all galaxies. At low metallicity it is common to deduce the electron temperature, $T_{\mathrm{e}}$, from the [O III $] 4363 /[\mathrm{O}$ III $] 4959,5007$ ratio and derive the oxygen abundance from the [OII] and [O III] lines - the so-called "direct" method or $T_{\mathrm{e}}$ method. At higher metallicity one might use one of several "strong-line" methods which are calibrated on photoionisation models (e.g. Osterbrock 1989; Charlot \& Longhetti 2001; Kewley \& Dopita 2002). However it is now well-known that there is a systematic offset between strong-line method and the $T_{\mathrm{e}}$ estimates of oxygen abundance (e.g. Kennicutt et al. 2000, 2003; Pilyugin 2000; Bresolin 2007). Thus there is no generally accepted method to estimate oxygen abundances for all abundance regimes and significant differences can be found between methods as discussed in detail by Kewley \& Ellison (2008).

We therefore tabulate a number of different abundance estimators for our objects to allow us to check how our results depend on the chosen estimator, we have adopted one representative of the three main classes of estimators, the direct method, the strong-line method and the empirically calibrated estimators. For all low-metallicity objects with detected [O III] $\lambda 4363 \AA$, we use the $T_{\mathrm{e}}$ method based on the formulae presented by Izotov et al. (2006) to estimate element abundances. For all galaxies we also calculate oxygen abundances using the Bayesian method discussed in T04 and B04; we will refer to these as CL01 abundances (a comparison with other methods is shown in T04). We 
also estimate oxygen abundances using the N2 method proposed by Pettini \& Pagel (2004, hereafter PP) and will refer to these as $\mathrm{PP}$ abundances in the following.

We have also verified that the results below do not change significantly if the PP O3N2 estimator, the $[\mathrm{N} \mathrm{II}] /[\mathrm{O}$ II] estimator advocated by Kewley \& Dopita (2002) or the P-method devised by Pilyugin (2000) are used instead, although slight changes do occur.

Unless otherwise stated we will in the following adopt $T_{\mathrm{e}}$ abundances for low-metallicity systems and CL01 abundances for the rest as a reference estimate. Specifically we adopt $T_{\mathrm{e}}$ estimators when they give $\mathrm{O} / \mathrm{H}<8.3$ and the $S / N$ in [O III]4363 is $>5$. Otherwise we assign the CL01 oxygen abundances adjusted by subtracting off a linearly varying offset which is -0.05 at $12+\log \mathrm{O} / \mathrm{H}=8.2$ and $0.3 \mathrm{dex}$ at $12+\log \mathrm{O} / \mathrm{H}=8.5$. We will refer to this as the mixed abundance estimator.

We arrived at this simple correction by comparing CL01 abundances for the HII regions in Kennicutt et al. (2003) with $T_{\mathrm{e}}$ abundances, requiring a continuous mass-metallicity relation and also taking into account the offsets seen in the comparison with $T_{\mathrm{e}}$ estimators by Yin et al. (2007).

\section{Identifying Wolf-Rayet features}

The main Wolf-Rayet features seen in the optical spectra of galaxies are two broad emission features: the blue bump around 4600-4680 $\AA$ and the red bump around 5650-5800 A. As mentioned in the introduction, the blue bump is primarily composed of lines from N III, N V, C III/IV and a broad He II emission line at $4686 \AA$.

These broad features do vary significantly in appearance, reflecting the strong variation in the relative emission line strength and velocity broadening seen in individual Wolf-Rayet stars. As a consequence the accurate measurement of the flux in WR bumps requires some care. Furthermore a number of nebular emission lines are often superposed on the Wolf-Rayet features making the disentangling of the individual fluxes rather challenging.

To identify candidate Wolf-Rayet galaxies we calculate the excess flux above the best-fit continuum in regions around the main Wolf-Rayet features. The features are typically weak and depend sensitively on the continuum estimation so a visual inspection is a necessary final step in this procedure to deal with false positives. However, it is necessary to have a semiautomated method to identify candidate Wolf-Rayet features in large, $N>10^{5}$, spectroscopic surveys.

Our approach is to first estimate the continuum doing a non-negative least-squares combination of single burst template spectra from the Bruzual \& Charlot (2003, BC03) library. We use a special version provided by Bruzual \& Charlot (priv. comm.), updated with empirical stellar spectra from the MILES spectral library (Sánchez-Blázquez et al. 2006). The method is essentially the same as that used in T04 and B04 but no smooth component is added to the spectral fit as this would often obliterate any signs of a Wolf-Rayet component. We note that this procedure is sensitive to the library of stellar spectra used. In particular the BC03 models with the STELIB library (Le Borgne et al. 2003) do show residual calibration features around $\mathrm{H} \beta$ (also noted by Asari et al. 2007), which serve to introduce features close to the expected location of the WR bumps and hence severely increase the number of false positives.
Table 1. The WR filters used for spectrophotometry in this work.

\begin{tabular}{lcc}
\hline \hline \multicolumn{1}{c}{ Name } & Central wavelength $(\AA)$ & Width $(\AA)$ \\
\hline He II & 4705 & 100 \\
He II continuum & 4517 & 50 \\
& 4785 & 50 \\
C IV & 5810 & 100 \\
C IV continuum & 5675 & 50 \\
& 5945 & 50 \\
\hline
\end{tabular}

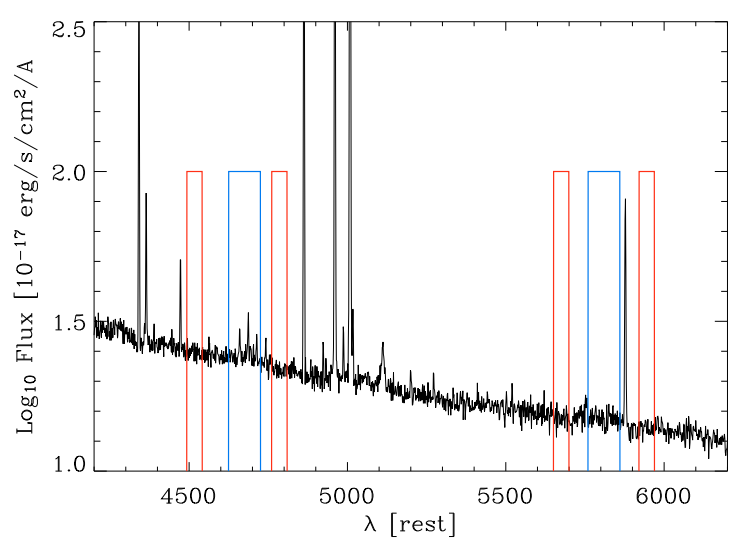

Fig. 1. The filter response functions adopted in this work.

From the continuum subtracted spectrum we define the excess in the blue WR feature around He II4686 (the blue bump) as

$e_{\text {blue }}=F_{\text {He II }}-F_{\text {He II continuum }}$

where $F$ is the summed flux in windows around the blue bump, corrected for nebular emission. We do a similar calculation for the red bump but have not used this for ranking.

The filters are square and are summarised in Table 1 and illustrated in Fig. 1. The red lines show the two continuum filters and the blue the "bump" filters. Note that the filter measuring the continuum consists of two parts to minimise the effect of a non-flat continuum. To determine the optimal filter functions we cross-correlated the Schaerer et al. (1999b, hereafter S99) catalogue with the SDSS DR6 and used those galaxies where the SDSS spectrum showed clear Wolf-Rayet features to determine what filter functions give us the best discrimination between WR and non-WR galaxies. For each filter we calculate $F$ by summing up the flux within the filter range of the spectrum after subtracting the continuum and nebular emission lines. We estimate the uncertainty on this flux by adding the uncertainty estimates of the spectrum in quadrature.

This results in a list of galaxies with possible Wolf-Rayet features and is similar to the approach used to identify WolfRayet stars in imaging surveys (e.g. Armandroff \& Massey 1985; Crowther et al. 2004). We emphasise that this approach is only used to find candidate WR galaxies.

Due to the large parent sample, the number of galaxies with elevated $e_{\text {blue }}$ due to noise, residual sky lines, instrumental failures and poor continuum fitting is comparable to or even higher than the number of genuine WR galaxies. As a consequence we have therefore carefully examined 11241 spectra sorted according to $e_{\text {blue }}$. These were assigned to four basic classes based on the appearance of the blue bump:

Class 3 Very clear Wolf-Rayet features. Typically a broad component to He II $\lambda 4686$ as well as N III $\lambda 4640$ are seen. 
Class 2 Convincing Wolf-Rayet features seen, but either noisier or not obvious before continuum subtraction.

Class 1 Possible Wolf-Rayet features but generally too noisy or too dependent on the continuum subtraction to be useable. Galaxies that show a single, apparently broad, He II $\lambda 4686$ line but no further Wolf-Rayet features are normally assigned to this class unless a clear identification of a broad component can be done.

Class 0 No Wolf-Rayet features seen.

In the following we will consider Classes 2 and 3 as being galaxies with Wolf-Rayet features and except for Sect. 5 below we will not distinguish between these two classes.

There are two crucial issues that must be taken into account when classifying the spectra visually. Firstly the sensitivity of the Wolf-Rayet features to the continuum fitting must be assessed for weak features. We do this by redoing the continuum fit using different wavelength ranges - typically contrasting a fit using 3800-7000 $\AA$ with one using only the spectrum between $4000 \AA$ and $5000 \AA$. If a feature changes significantly between these two continuum fits it is normally assigned Class 0 . The other problematic issue has to do with nebular emission. This is discussed in detail in the following section, but for the visual classification the main problem is when a nebular He II $\lambda 4686$ line is superposed on a broader line. The SDSS spectra often do not have sufficiently high $S / N$ to disentangle these two components, and unless other supporting features such as N III $\lambda 4640$ can be seen, the galaxy will be assigned to either Class 1 or Class 0 , depending on the strength of the broad component as judged by the rigourous fits described below.

\section{Fitting Wolf-Rayet features}

The existing literature on Wolf-Rayet galaxies usually estimates the bump luminosity by fitting a single Gaussian to the spectrum after removal of emission lines, either subtracted or masked (e.g. Guseva et al. 2000). This has been an acceptable approach because the resolution of the spectra have often been fairly low. With the SDSS spectra it is clear that this is not always a good solution as a single Gaussian often provides a poor fit to the overall detected features. This was also realised by Zhang et al. (2007) in their study of WR galaxies in the SDSS and they therefore calculated the flux integrating all the flux between $4600 \AA$ and $4750 \AA$ after correction for nebular emission. This does remove all distinction of $\mathrm{WN}$ and $\mathrm{WC}$ features however, and it is not an optimal approach in terms of $S / N$ since regions with no WR features are included in the calculation. Finally, the subtraction of nebular lines cannot be viewed as a separate problem from fitting the broad features as there are significant degeneracies between these.

We have therefore adopted a more rigourous approach: we fit the relevant nebular emission lines jointly with Gaussians for each feature which can be expected to be present based on the observations of WR stars in the local Universe. In the blue we fit:

1. Nebular lines: [Fe III] $\lambda 4559$, [Fe III] $\lambda 4669$, He II $\lambda 4686$, [Fe III] $\lambda 4702$, [Ar IV] $\lambda 4711$, He I $\lambda$ 4714. The line width is fixed to that determined from the joint fit to the strong emission lines in the spectrum. The [Ar IV] $\lambda 4740$ line is far enough from the features of interest that we do not need to include it in the fit.

2. Wolf-Rayet features: the blue bump is primarily composed of N V, N III, C III/IV blends as well as the He II $\lambda 4686$ line. The N V doublet is fit as a single feature centred at $4610 \AA$.

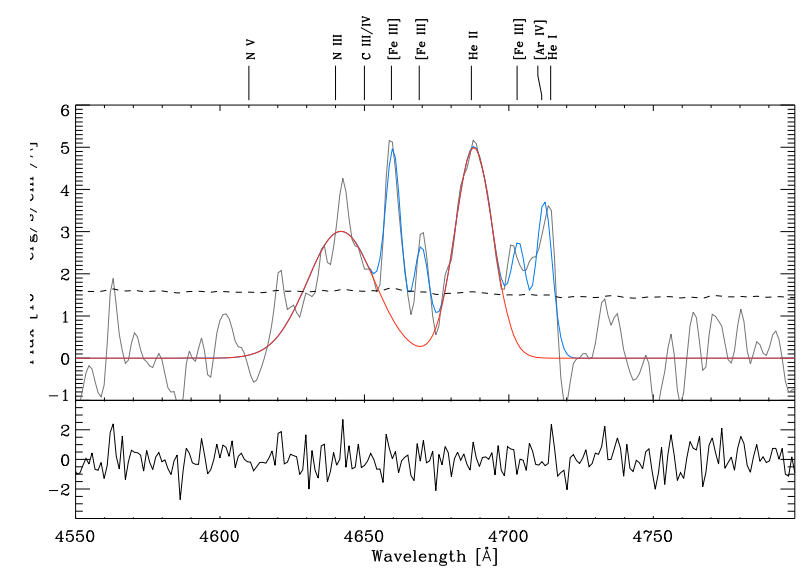

Fig. 2. An example of a fit to the blue bump for a WR galaxy. The solid black line shows the continuum subtracted spectrum smoothed with a $3 \AA$ Gaussian. The dashed black line shows the noise level assuming uncorrelated errors in the pixels. The red line shows the fit to the blue bump features. In this particular fit there is a N III component as well as a broad component to He II. The blue line shows the narrow emission lines fitted in this spectrum. The bottom panel shows the residual after subtracting the best fit model from the spectrum, normalised by the uncertainty estimate in each pixel. This residual spectrum is consistent with pure Gaussian noise.

The N III feature is centred around $4640 \AA$, whereas the C III/IV feature is assumed to be centred at $4650 \AA$ and a broad component to He II $\lambda 4686$ is also included in the fit.

An example of a blue bump fit is shown in Fig. 2. The red line traces the WR features fit, whereas the blue line shows the nebular emission lines that must be included in the fit. When working with this sample it became evident that there were several [Fe III] lines that normally are not taken into account, particularly at $4669 \AA$ and $4702 \AA$ which will bias any fits to the bump features high if not removed. It is also obvious from this figure that a single Gaussian is a poor approximation to the bump shape.

In the red there are fewer nebular emission lines superposed on the broad WR features and we fit:

1. nebular emission lines: [N II] $\lambda 5755$ and He I $\lambda 5876$;

2. Wolf-Rayet features: one feature centred at $5696 \AA$ is fit as well as a doublet at 5803, $5815 \AA$.

When carrying out the fit, the line fluxes are constrained to be non-negative. This might cause a slight bias in the derived fluxes at very low flux values, but was found to be required to ensure physically meaningful fits. We furthermore limit the overall wavelength shift of the blue and red features to be $|\Delta \lambda|<3 \AA$. This has no influence on the results.

However we also found that we needed to provide an upper limit to the width of the Wolf-Rayet features. This upper limit does affect the relative contributions of the different features to the overall bump although the integrated flux is not significantly changed. We have generally adopted $20 \AA$ (FWHM $\sim 47 \AA)$ to ensure an adequate fit. This corresponds to $\sigma \sim 1280 \mathrm{~km} \mathrm{~s}^{-1}$ $\left(\sim 3000 \mathrm{~km} \mathrm{~s}^{-1} F W H M\right)$ and we have found this to be a reasonable default upper limit to the width of the individual Wolf-Rayet features for all our galaxies. For reference only 2 stars in the compilation of Hadfield \& Crowther (2006) exceed this velocity width. All fits were visually inspected and for 58 we redid the fit manually to better reproduce the shape of the bump. Figure 4 shows a collection of spectra ordered by increasing $E W(\mathrm{H} \beta)$. It 


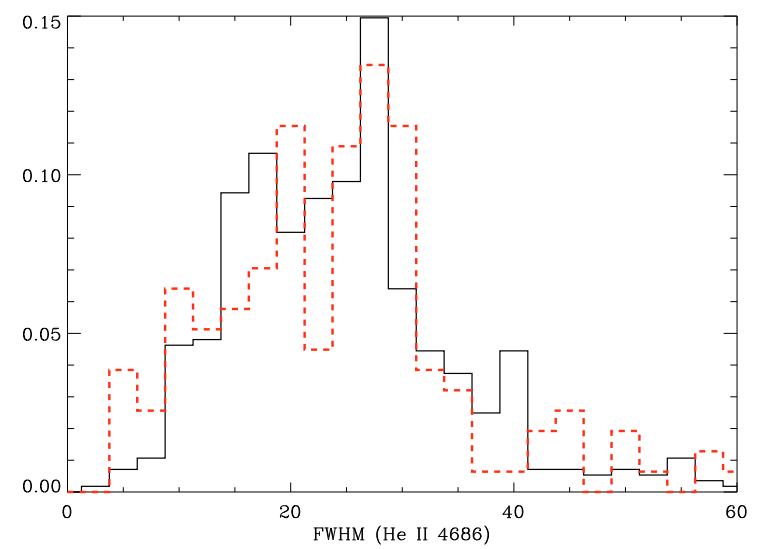

Fig. 3. The distribution of widths of the He II $\lambda 4686$ line measured in Galactic, LMC and SMC WR stars (dashed line) compared to the widths determined by the fit to our Wolf-Rayet candidate galaxies (solid line).

is noticeable that the bumps show a range of morphologies reflecting the broad mix of WN and WC stars seen in these spectra.

We can test these fits in a model independent manner by realising that the intrinsic line widths of the Wolf-Rayet features are considerably larger than those caused by galactic rotation, so we can reasonably assume that the distribution of the $F W H M$ of the He II $\lambda 4686$ line measured by our fits should follow closely the distribution found for Galactic, LMC and SMC Wolf-Rayet stars $^{1}$. To do this we combined the measurements for Galactic and LMC WN stars in Smith et al. (1996) and for the LMC and SMC in Crowther \& Hadfield (2006). Where two measurements of the same star existed we took a straight average.

The results of this comparison are shown in Fig. 3. Clearly there is very good agreement between the two distributions and this can be taken as a reasonable consistency check. It also indicates that we can make use of the width of the He II feature to study the variation of the width of WR features with galaxy properties.

Note that we do seem to have fewer narrow He II features than seen in the stars. This is to be expected, both because the narrow features are more difficult to disentangle from the nebular lines, and because when averaged over a population the lines tend to be broadened due to peculiar motions and because on average the WR stars with broad lines appear to be more luminous (CH06).

To ascertain the reliability of the deblending of the individual features in the blue we carried out a number of simulations where we added noise to a set of realistic simulated spectra with Wolf-Rayet features superposed. This showed that the fits have significant degeneracies between the individual features, in particular between the N III and C III/IV features which are closely spaced, but also with the superposed nebular lines. For the purposes of this paper we will mostly make use of the total blue and red bump fluxes rather than the individual features. This does limit our comparisons to models somewhat but it will turn out to be sufficient for the purposes of this paper.

The procedure adopted for classification of WR galaxies has not made any explicit cuts on the equivalent width or $S / N$ of the bump features. Figure 5 shows the resulting distribution of the equivalent width of the blue bump. This shows that the spectra

\footnotetext{
1 These three samples of WR stars sample reasonably well the overall metallicity distribution of our WR galaxies so this comparison should be meaningful.
}

have an effective minimum $E W$ of $1 \AA$, and this is also constant with redshift. It is therefore reasonable to take this as the a posteriori completeness limit for our sample.

\section{Overall sample properties}

\subsection{Spectral classification and overall inventory}

We follow B04 in separating the galaxies in our sample based on their location in the Baldwin et al. (1981, BPT) ionisation diagram. We will refer to galaxies whose emission line spectrum is dominated by star formation as the SF class, and to those dominated by an active galactic nucleus (AGN) as the AGN class. Galaxies that fall between these two classes are assigned to the Composite class, see B04 for details.

As discussed in B04 (see also e.g. T04; Kauffmann et al. 2003a; Kewley et al. 2006) the reliable classification of emission line galaxies requires a $S / N$ in each of the lines $\mathrm{H} \beta$, [O III] $\lambda 5007, \mathrm{H} \alpha$ and [N II] $\lambda 6584$ of at least $S / N=3$. For a few galaxies the [O III] $\lambda 5007$ line is truncated by the SDSS pipeline reductions; for these [O III] $\lambda 4959$ is used instead. Finally a few galaxies have no [N II] $\lambda 6584$ detected at $S / N>3$ and for classification purposes and abundance determinations we assign these the $3 \sigma$ upper limit to the line flux. After these steps a total of $9500(3.1 \%)$ galaxies cannot be classified using emission line diagnostic and are placed in the Unclassified category. For galaxies with $E W(\mathrm{H} \beta)>5 \AA$, this is entirely due to instrumental problems and we have verified that this does not lead to any systematic biases between the parent sample and the WR galaxy sample. For the galaxies with weaker $\mathrm{H} \beta$ there will be a trend that the fraction of unclassifiable galaxies increases from $\sim 4 \%$ at $E W(\mathrm{H} \beta)=7 \AA$ to $\sim 50 \%$ at $E W(\mathrm{H} \beta)=2 \AA$. Hence any comparisons of the abundance of WR galaxies with the parent population at low equivalent widths must take this into account if appropriate.

The final sample is summarised in Table 2 and given in full Table 3 which is available online. The luminosities and equivalent widths of the blue and red bumps as well as the width of the Gaussian fit to the He II $\lambda 4686$ line are given in Table 4 and the abundances derived from the spectra in Table 5. The full sample consists of 570 spectra from the SDSS. This is more than twice the number of all previously known Wolf-Rayet galaxies, and a factor of $\sim 3$ more than the largest rigourously selected sample of WR galaxies from a single study, surpassing the 174 galaxies in the Zhang et al. (2007) study. We should comment that in fact only 101 of the objects from that survey are classified as class 2 or 3 by us and a further 36 as class 1 . For the final 37 we are unable to reproduce the identification of broad Wolf-Rayet features by Zhang et al.

Out of the 570 spectra, a total of 531 have SCIENCEPRIMARY $=1$ and can thus be used for statistical studies. A total of 419 of these have $z>0.005$ which we will occasionally adopt as our lower redshift limit because deviations from the Hubble flow are limited and the photometry from the SDSS is more reliable (see Blanton et al. 2005) at these redshifts.

Combining our sample with the compilation of S99 leads to a total of 641 unique Wolf-Rayet galaxies, including galaxies classified as Class 1 would increase this to 1613 unique sources, a total of 1778 spectra. To put this in perspective it is worth pointing out that only 227 Wolf-Rayet stars in the Milky Way are listed in the compilation by van der Hucht (2001), and with 135 stars in the LMC and 9 in the SMC listed by van der Hucht, the total sample of Wolf-Rayet galaxies here is comparable in 

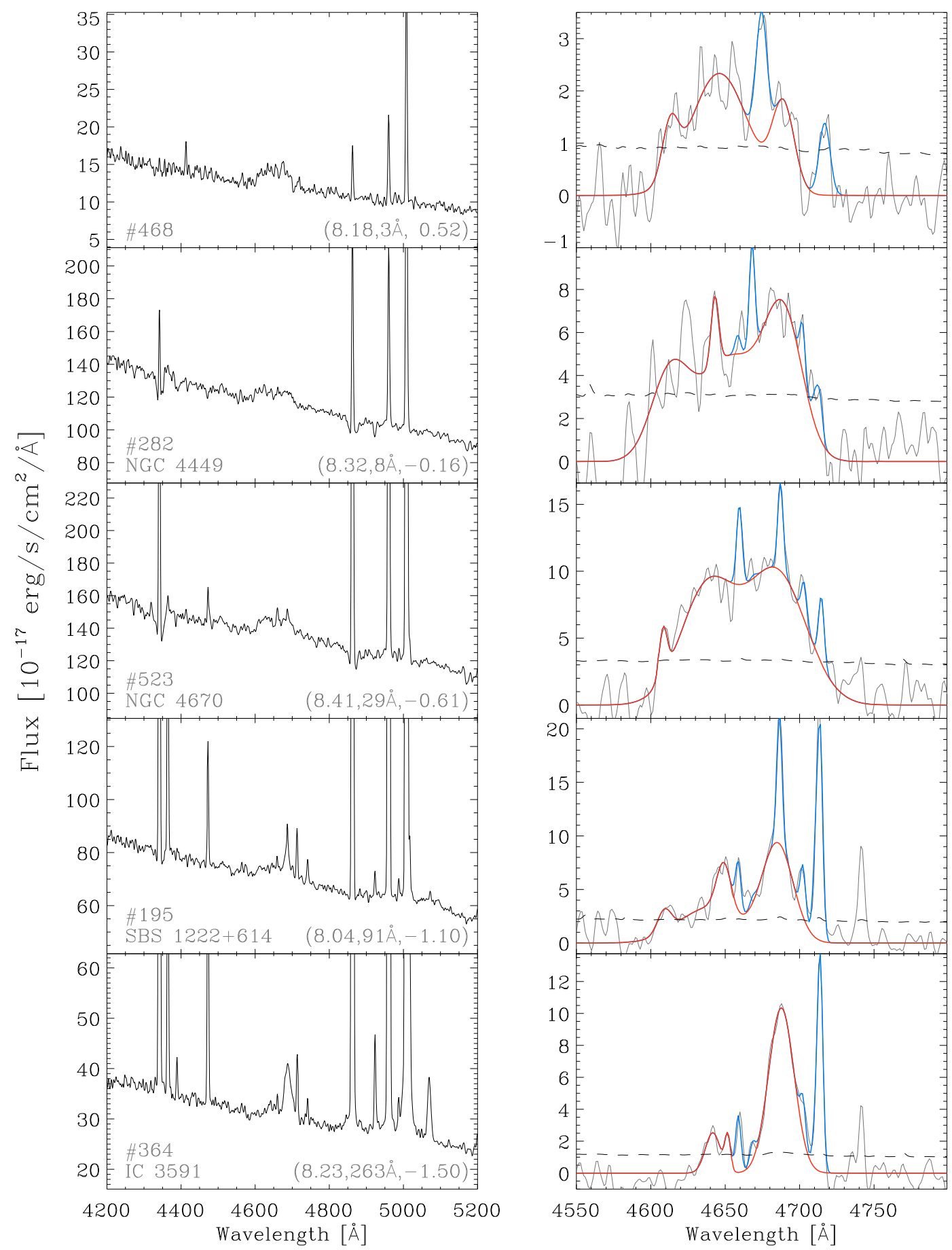

Fig. 4. A montage of five WR galaxy spectra ordered by $E W(\mathrm{H} \beta)$ increasing by a factor of 2 downwards. The left column shows the full spectrum zoomed in around the blue bump and the right column the continuum subtracted spectrum similarly to that shown in Fig. 2. The number of the spectrum in our catalogue as well as any common names are indicated in the lower left corner of the full spectrum plot. The three numbers in the parenthesis in the lower right corner show $12+\log \mathrm{O} / \mathrm{H}, E W(\mathrm{H} \beta)$ and $\log L(\mathrm{Blue}$ bump $) / L(\mathrm{H} \beta)$.

size to the samples of individual Wolf-Rayet stars studied in detail. Thus we expect that the present study will provide a useful complement to the studies of individual stars in nearby galaxies.

\subsection{Physical properties of the host galaxies}

Figure 6 shows the redshift distribution of the SDSS parent sample as the shaded grey histogram in the top left panel. The bins each contain 5000 galaxies and the histogram is normalised to have the same peak value as the black histogram. The redshift distributions of Class 2 and Class 3 objects together with the redshift distribution of the two taken together are superimposed. What is obvious is that the galaxies showing Wolf-Rayet features have a redshift distribution that is strongly shifted towards low redshift compared with the SDSS as a whole. This is natural because more distant galaxies must be intrinsically more luminous to fall within the selection limits of the SDSS and this makes the detection of Wolf-Rayet features more difficult.

This is more explicitly illustrated in the top right panel which shows the percentage of all galaxies in the SDSS that show 


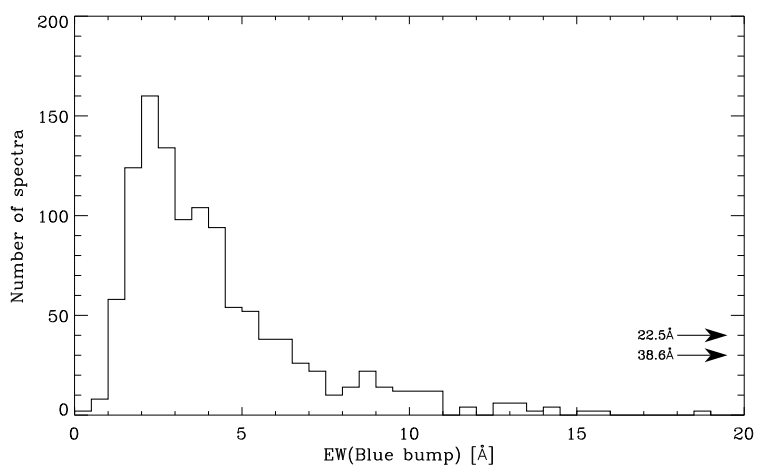

Fig. 5. The distribution of the equivalent width of the blue bump measured four our Class 2 and Class 3 spectra. Note the strong drop at $E W<1 \AA$. Two spectra fall outside the plotted region as indicated by arrows.

Table 2. The summary of the Wolf-Rayet sample.

\begin{tabular}{lrr}
\hline \hline Sub-sample & Number & Number \\
& All $z$ & $0.005<z<0.22$ \\
\hline Class 2+3 & 570 & 452 \\
Class 2 & 373 & 317 \\
Class 3 & 197 & 135 \\
Class 1 & 1115 & 1058 \\
Star-forming & 453 & 346 \\
AGN & 42 & 38 \\
Composite & 68 & 67 \\
Unclassified & 7 & 1 \\
\hline \multicolumn{2}{c}{ With SCIENCEPRIMARY=1 } \\
\hline Class 2+3 & 531 & 419 \\
Class 2 & 344 & 293 \\
Class 3 & 187 & 126 \\
Class 1 & 972 & 919 \\
Star-forming & 422 & 321 \\
AGN & 38 & 34 \\
Composite & 64 & 63 \\
Unclassified & 7 & 1 \\
\hline
\end{tabular}

Wolf-Rayet features as a function of redshift. The bins here are chosen to contain 30 galaxies with Wolf-Rayet features each. Clearly galaxies with Wolf-Rayet features make up a significant number of the very low $z$ spectra in the SDSS.

The middle row shows the same information but the distributions are now those of the dust-sensitive $\mathrm{H} \alpha / \mathrm{H} \beta$ ratio and only galaxies that fall within the SF class are included because it is only for these galaxies that the $\mathrm{H} \alpha / \mathrm{H} \beta$ ratio can be easily interpreted in terms of dust attenuation. It is clear that WolfRayet galaxies typically have lower dust extinction than typical SDSS galaxies.

Finally the bottom row shows the distributions against the concentration parameter, $R_{90} / R_{50}$, where $R_{50}$ and $R_{90}$ are the radii that enclose $50 \%$ and $90 \%$ of the light respectively out to the Petrosian radius of the galaxies. This is known to correlate with visual morphology (Shimasaku et al. 2001). It is noteworthy that galaxies with Wolf-Rayet features appear to have a very broad range in morphology (right-hand panel) from the most diffuse to the most concentrated galaxies.

It is reassuring that in the plot for $\mathrm{H} \alpha / \mathrm{H} \beta$ and concentration, the distributions of Class 2 and Class 3 sources are very similar, as one would expect since these quantities ought not to strongly affect the detectability of Wolf-Rayet features.

Figure 7 shows the location of WR galaxies in $g-r$ colour in the top row. As is clear from the right-hand panel, the fraction
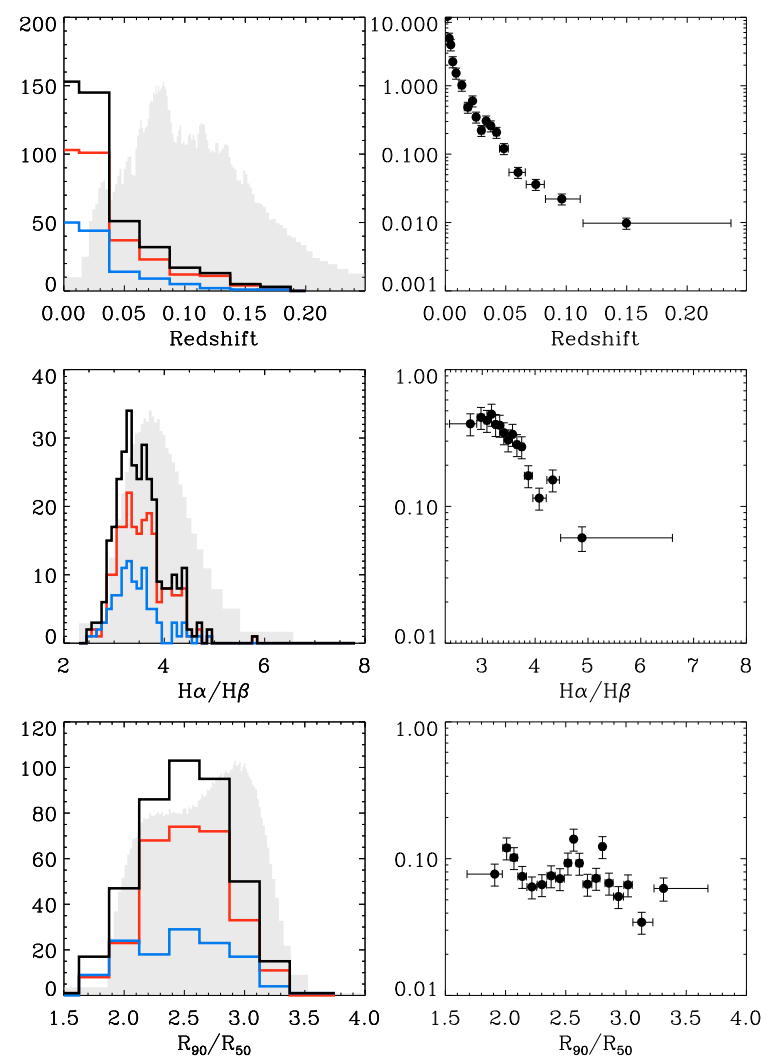

Fig. 6. The overall properties of the Wolf-Rayet candidate galaxies compared with the properties of the SDSS parent sample as a whole (shaded grey histograms) - duplicates have been excluded. The top row shows the redshift distribution of the SDSS as a filled light-grey histogram on the left, with the distribution of Class 3 objects in blue, Class 2 objects in red and the two classes combined as the black overplotted histogram. The right hand panel shows the percentage of galaxies at a given redshift that show Wolf-Rayet features. The second row shows the same but for the dust sensitive $\mathrm{H} \alpha / \mathrm{H} \beta$ ratio and the bottom row the concentration parameter.

of galaxies that show Wolf-Rayet features drops precipitously at $g-r>0$. This $g-r$ colour is calculated from the SDSS model colours with no $k$-correction and it has been corrected from emission line contamination assuming that the contribution from emission line flux found within the fibre is appropriate for the galaxy as a whole.

The middle row of Fig. 7 shows the distribution of $E W(\mathrm{H} \beta)$. It is readily seen that the fraction of galaxies with Wolf-Rayet features increases rapidly with $E W(\mathrm{H} \beta)$, reaching an apparent plateau with nearly $20 \%$ of galaxies with $E W(\mathrm{H} \beta)>100 \AA$ showing Wolf-Rayet bumps.

The bottom panel shows the distribution with respect to metallicity for the full sample. This includes only the SF class objects. It shows that a smaller fraction of galaxies with high metallicity shows Wolf-Rayet features than objects with lower metallicity, although we should caution that this particular plot does depend on how the metallicities of the objects are assigned. At first glance this might appear to say that Wolf-Rayet stars are less common at high metallicity, but that ignores the multivariate nature of the present sample, and we address the interesting question of the abundance of Wolf-Rayet stars at different metallicities using a multi-dimensional view in Sect. 6 below.

In addition to the preceding discussion which places WolfRayet galaxies in the context of the overall galaxy population, it is also of interest to ask whether there are systematic 

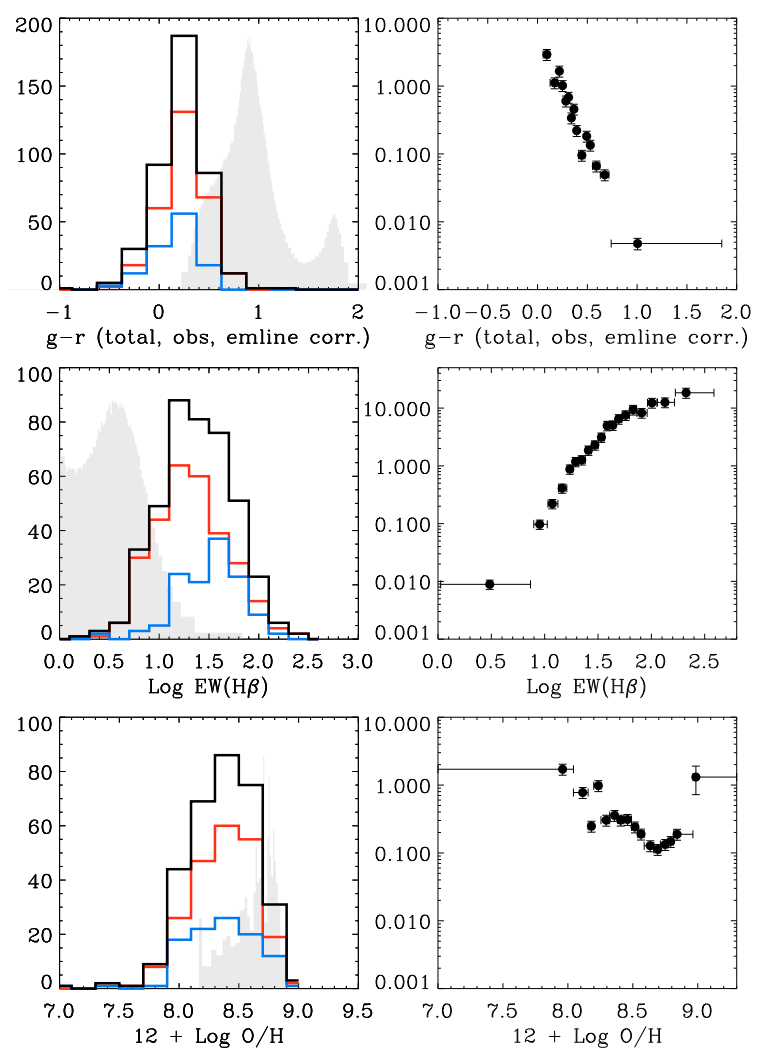

Fig. 7. Similar to Fig. 6. The top row shows the total $g-r$ colour, corrected for the emission line contribution to the flux as discussed in the text. The middle row shows the $E W(\mathrm{H} \beta)$ and the bottom row the oxygen abundance - note that only galaxies classified as SF are included in the final panel.

differences between galaxies showing Wolf-Rayet features and similar galaxies without WR features. The bottom line of this exercise is that WR galaxies and similar galaxies without WR features do not differ in dust attenuation but that there is a slight tendency for WR galaxies to be more concentrated than galaxies with similar mass and star formation activity without WR features.

To reach these conclusions we adopted a similar approach to Kauffmann et al. (2006). For each WR galaxy we find all SDSS galaxies whose stellar mass is within 0.1 dex of the WR galaxy, the $\mathrm{D} 4000_{N}$ is within 0.05 and $E W(\mathrm{H} \beta)$ is within a factor of 3 of that of the WR galaxy. If any of these cuts select less than 1000 galaxies we loosen the constraints to the first 1000 objects. We then calculate the difference between the properties of the WR galaxies and these similar SDSS galaxies.

\subsection{Host galaxy morphology and location of WR regions}

As mentioned above a number of the spectra studied here are those of non-nuclear regions in the galaxies. This is the case for approximately $30 \%$ of the spectra, the rest correspond to the central regions of the galaxies. In the case of mergers and some types of irregular galaxies it is difficult to determine a centre, this is true for $\sim 5 \%$ of the spectra. However most spectra do originate well within the main body of the galaxies, only 93 spectra (16\%) really originate from the outskirts of the host galaxy. These are invariably bright $\mathrm{H}$ II-regions, in fact a total of 202 (35\%) of the spectra can be identified with a clearly delimited star forming region, ranging from $\mathrm{H}$ II-regions to massive super-star clusters spanning almost $50 \%$ of the galaxy.

The host galaxies span a range of morphologies and dynamical situations. There are a number of clear mergers, at least $7 \%$ of the spectra, and $\sim 11 \%$ originate from galaxies with a strong bar. Thus one might worry that these classes have star bursts of different effective ages and hence that including them all in one group might bias or wash-out any trends. To check this we have tested a large number of relationships and seen how they vary with the location of galaxies and with the morphological properties of the galaxies. We do find that oxygen abundance distribution of spectra originating in the outskirts of their parent galaxies are slightly shifted to lower metallicity as compared to that for the central region, as one might expect given the wellknown metallicity gradients in galaxies. Apart from this there are no detectable dependencies on any relationships/trends with the regions of the galaxies the spectra originate from or with the galaxy morphologies. We will therefore ignore this in the following.

\section{The abundance of WR stars with metallicity}

Models for WR star formation predict that the number of WolfRayet stars increases with increasing metallicity (Meynet \& Maeder 2005) due in large part to a strong metallicity dependence on the stellar winds (Vink \& de Koter 2005). From an observational point of view it is also established that there is a decline in the number of WR stars as compared to O-stars, $\mathrm{N}(\mathrm{WR}) / \mathrm{N}(\mathrm{O})$, with declining metallicity (see Crowther 2007, for an overview). However, the range of metallicities is limited and the spread around the relation is not established and the stellar parameters are typically hard to constrain without involving modelling (e.g. Hamann et al. 2006). In this section we will focus on empirical measures of the abundance of WR stars, postponing a comparison with models to Sect. 10.

Figure 8 shows the fraction of SF galaxies showing WR features as a function of oxygen abundance in four bins in $E W(\mathrm{H} \beta)$. It shows a very clear increase in the fraction of galaxies showing WR features with metallicity with a clear indication of a non-linear relationship. The comparison is done in bins of $E W(\mathrm{H} \beta)$ to take out the correlation between oxygen abundance and $E W(\mathrm{H} \beta)$ in the SDSS sample. It is, however, clear that the trend is similar for different bins in $E W(\mathrm{H} \beta)$ and it turns out that they are independent of the abundance estimator chosen.

The figure shows a clear relationship between the oxygen abundance of a galaxy and the likelihood that it harbours significant numbers of Wolf-Rayet stars. It is also clear that there is a correlation with $E W(\mathrm{H} \beta)$. This is made more explicit in Fig. 9 which shows the relative abundance of WR galaxies as a function of $E W(\mathrm{H} \beta)$ in four bins of oxygen abundance. It is clear that galaxies with the same $E W(\mathrm{H} \beta)$ are more likely to have WR features at higher metallicity.

It is also intriguing that all the curves appear to reach a maximum value and then turn over. This is expected to happen when the $E W(\mathrm{H} \beta)$ samples burst ages that are short relative to the time of start of the WR phase (after $\sim 2 \mathrm{Myr}$ ). While $E W(\mathrm{H} \beta)$ is often used as an age indicator (Copetti et al. 1986; Zhang et al. 2007) it is well-known that this might be a poor approximation both due to different dust attenuation of the lines and the continuum and to an older underlying population (e.g. Mas-Hesse \& Kunth 1999). For our sample this is means that $E W(\mathrm{H} \beta)$ in general is not a reliable age indicator, but for a given metallicity range it might be useful as a relative age indicator and/or burst strength indicator. The fraction appears to reach a maximum at 


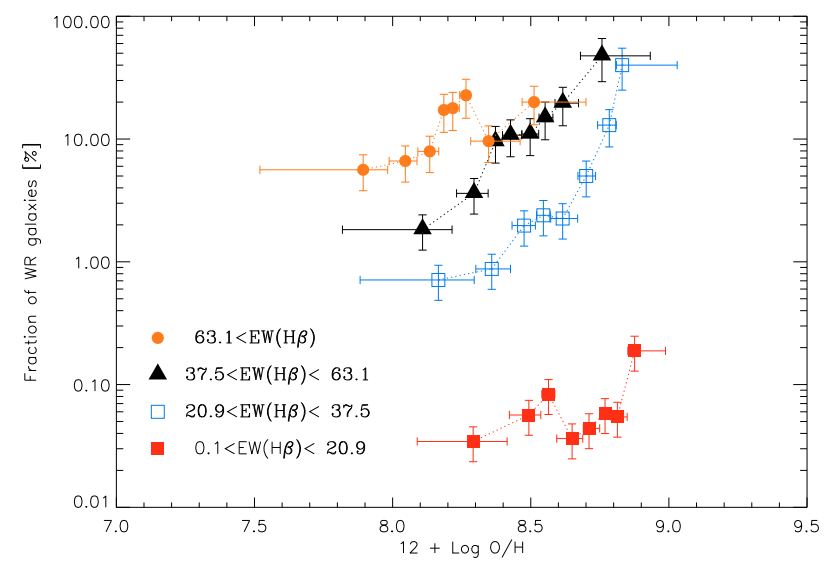

Fig. 8. The fraction of SF galaxies that show Wolf-Rayet features as a function of oxygen abundance in four different $E W(\mathrm{H} \beta)$ bins. The bins in $E W(\mathrm{H} \beta)$ each contain 80 Class 2 and 3 galaxies, except the highest bin which contains 81 . The points are calculated in bins containing 10 Class 2 and 3 galaxies each and the error bars reflect Poissonian uncertainties. Only SF galaxies are included and the oxygen abundance estimator is the mixed one.

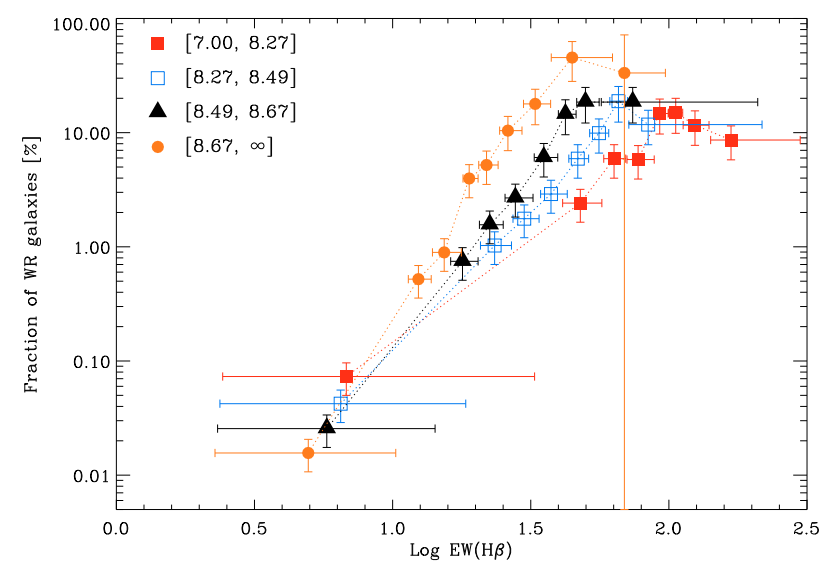

Fig. 9. Similar to Fig. 8, this figure shows the the fraction of SF galaxies that contain Wolf-Rayet features as a function of $E W(\mathrm{H} \beta)$ in four bins of oxygen abundance.

$\log E W(\mathrm{H} \beta)>1.7$. For a short ( $<1 \mathrm{Myr}$ ) burst this corresponds to an age of 5-6 Myr. Since 10-20\% of all spectra at this $E W$ show WR features, we conclude that the WR features are clearly visible in $0.5-1 \mathrm{Myr}$ of this time, although we should caution that the age estimates are strongly dependent on the assumed properties of the burst.

An alternative formulation of this result can be found by adopting the method already used by Kunth \& Sargent (1981) to determine the ratio of WR to O stars (see also Zhang et al. 2007). This requires a comparison between metal content and the flux of the blue bump divided by the $\mathrm{H} \beta$ flux. This is a rough, but model independent, estimate of the number of WR stars per O stars. This follows because to first order, the flux in the blue bump is proportional to the number of WR stars. In addition the luminosity of the $\mathrm{H} \beta$ line is roughly proportional to the number of $\mathrm{O}$ stars within the area surveyed and the ratio is approximately independent of dust attenuation (see discussion in Sect. 2.2). Thus this quantity should give a reasonable estimate of the relative number of WR and $\mathrm{O}$ stars in a model-independent way. It has recently become common to convert this to $N(\mathrm{WR}) / N(\mathrm{O}+\mathrm{WR})$ in a model dependent way (e.g. Guseva et al. 2000; Fernandes et al. 2004) but in view of the uncertainties in the current models

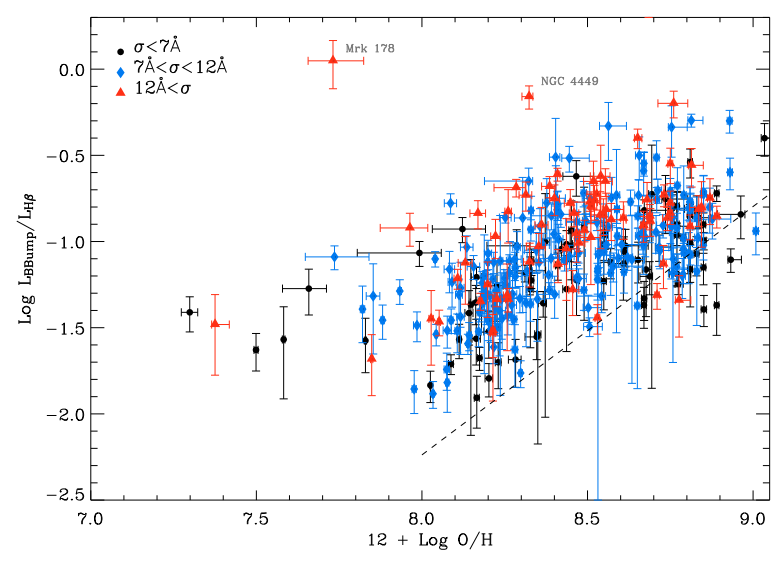

Fig. 10. The ratio of the blue bump luminosity to the luminosity of the $\mathrm{H} \beta$ as a function of metallicity. There is a clear trend towards lower maximum bump luminosities relative to $\mathrm{H} \beta$ at lower metallicity but it appears to flatten out at $12+\log \mathrm{O} / \mathrm{H}<8$. The dashed line shows the detection limit based on the data in Fig. 5. The points are colour coded according to the width of the He II $\lambda 4686$ line. The red points show the location of the galaxies with the widest bumps, the black the narrowest. There is no statistically significant difference between these classes.

discussed below we prefer not to do this at this point, and return to it in Sect. 10.2 below.

Figure 10 shows the results of this exercise. The figure plots galaxy oxygen abundance along the $x$-axis. There is an apparent correlation between the metallicity and the ratio of the blue bump to $\mathrm{H} \beta$ luminosities but the lack of points in the bottom right is due to incompleteness. This is shown by the dashed line which indicates the location of a galaxy with an $E W$ (Blue bump) of $1 \AA$ given the observed distribution of $E W(\mathrm{H} \beta)$ at that metallicity in the full SDSS DR6. The spread of points at a given metallicity is several times the observational uncertainty and is entirely consistent with being due to the fact that the star-bursts are not all coeval.

However the decrease in the maximum value attained by the ratio of blue bump luminosity to $\mathrm{H} \beta$ luminosity is real and reflects a decrease in the total luminosity of the Wolf-Rayet phase with decreasing luminosity. This trend does however not extend to the very lowest metallicities and at metallicities below $12+\log \mathrm{O} / \mathrm{H}<8$ there appears to be a flattening off.

As mentioned by $\mathrm{CHO6}$ the WN stars in the SMC are known to have weaker and narrower lines than in more metal rich environments (Conti et al. 1989) so we also indicate by different symbols and colours the location of galaxies with different He II 4686 widths. There is no statistically significant difference between these classes but it is clear that the systems with the strongest bumps relative to $\mathrm{H} \beta$ all show broad He II lines. In particular the extreme galaxy Mrk 178 which has the strongest WR features relative to $\mathrm{H} \beta$ of any galaxy in the sample, shows broad WR lines and an intriguingly low metallicity. We will discuss this object in some detail in Sect. 10.

\section{The ionisation conditions of the interstellar medium of WR galaxies}

It is of considerable interest to understand the properties of the interstellar medium (ISM) of galaxies with very strong star formation activity since this might give important insights into the ISM of actively star forming galaxies at all redshifts (e.g. Brinchmann et al. 2008; Liu et al. 2008). 

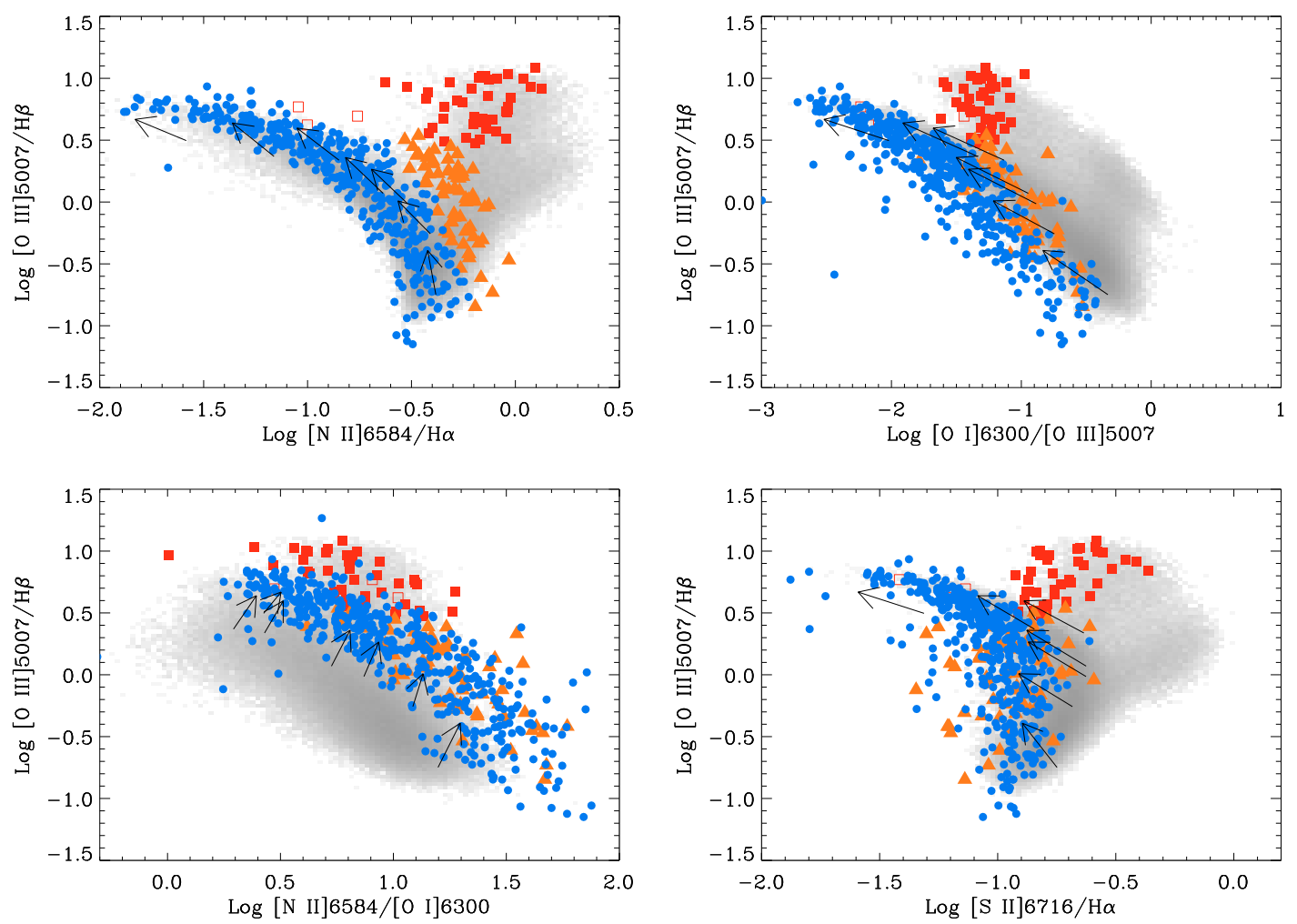

Fig. 11. Top left: the BPT diagram for the SDSS with Wolf-Rayet galaxies overplotted. The top right panel shows the $[\mathrm{O}$ III] $\lambda 5007 / \mathrm{H} \beta$ versus [O I] $\lambda 6300 /[\mathrm{O}$ III] $\lambda 5007$ diagram and the bottom left two further diagnostic diagrams. In all panels the grey-scale shows the logarithm of the number of galaxies in bins on the $\log [N$ II $] \lambda 6584 / \mathrm{H} \alpha$ vs. $\log [O \mathrm{III}] \lambda 5007 / \mathrm{H} \beta$ plane. The galaxies plotted are chosen to have $S / N>3$ in all these four lines as well as [O I] $\lambda 6300$ and [S II] $\lambda 6717$. The points show the location of our candidate WR galaxies. The blue filled circles show the galaxies classified as SF, the orange triangles that of the composite galaxies and the filled red squares the location of AGNs. The open red squares show the location of Composite and AGNs that have $\log [N$ II $] \lambda 6584 / \mathrm{H} \alpha<-0.7$. These objects resemble SF galaxies more than AGN. The small black arrows show the effect of increasing the ionisation parameter, $U$, by $0.5 \mathrm{dex}$, see text for details.

Here we ask whether galaxies that harbour significant populations of Wolf-Rayet stars differ systematically in their emission line properties from other star forming galaxies. We use the BPT diagram shown in the top-right panel of Fig. 11 as a starting point. This shows the distribution of all SDSS galaxies with $S / N>3$ in $\mathrm{H} \beta$, [O III] $\lambda 5007$, [O I] $\lambda 6300, \mathrm{H} \alpha$, [N II] $\lambda 6584$ and $[\mathrm{S} \mathrm{II}] \lambda 6717$ as a grey scale $2 \mathrm{D}$ distribution - the grey-scale shows the logarithm of the number of galaxies in each bin. The galaxies showing WR signatures are overplotted with different symbols indicating different emission line classifications with red squares indicating AGNs, orange triangles Composite objects and the filled blue circles Wolf-Rayet galaxies in the SF class. The open red squares indicate five galaxies lying well above the main sample of galaxies, in fact close to the location of the $z \sim 2$ galaxies studied by Erb et al. (2006). While these galaxies formally lie in the region of Composite or AGN galaxies, i.e. above the locus of photoionisation models for H II-regions (Kewley et al. 2001), some of these will turn out to show characteristics of star forming systems. Since it is well-known that photoionisation models tend to underpredict the [O III] $\lambda 5007 / \mathrm{H} \beta$ ratio for low metallicity $\mathrm{H}$ II-regions (e.g. Dopita et al. 2006), we have chosen to identify these objects with a different symbol in Fig. 11

The top right panel of Fig. 11 shows the [O III] $\lambda 5007 / \mathrm{H} \beta$ versus [OI] $\lambda 6300 /[\mathrm{O}$ III $] \lambda 5007$ ratio. While the AGN are distributed in a similar manner to the main bulk of AGN, the SF galaxies with WR signatures seem to be offset from the bulk of the SDSS galaxies. Since the [O I] $\lambda 6300$ line predominantly originates in the neutral ISM, this might indicate that the
WR galaxies have a significantly more ionised ISM compared to other SF galaxies.

This offset is even clearer in the bottom right panel, which has [N II] $\lambda 6584 /[\mathrm{O}$ I] $\lambda 6300$ on the $x$-axis. Clearly the SF galaxies showing WR signatures occupy almost the same region as the AGN and composite galaxies. This might be interpreted as a suppressed [O I] $\lambda 6300$ shifting the galaxies rightwards from the SF sequence.

A similar offset can be seen in the [O III $] \lambda 5007 / \mathrm{H} \beta$ versus [S II] $\lambda 6716 / \mathrm{H} \alpha$ diagram shown in the bottom right panel. [S II] is also significantly produced in the neutral ISM and it is again clear that there is a systematic offset between the WR galaxies and the bulk of galaxies in the SDSS.

On these diagrams we have indicated the effect of changing the ionisation parameter, $U$, by 0.5 dex by the black arrows, for six different metal abundances based on the CL01 models. It is clear that the offsets we see are consistent with a higher effective ionisation parameter in the Wolf-Rayet galaxies than in the SDSS as a whole. This is in good agreement with the findings of Brinchmann et al. (2008, BCP08) and Liu et al. (2008) for strongly star-forming galaxies in the SDSS in general - see BPC08 for more discussion. It is worth pointing out that such simple scaling arguments are insufficient to reliably distinguish between an increased ionisation parameter or a non-negligible escape of ionising photons from the region sampled by the spectrum (e.g. Binette et al. 1996). A more thorough analysis would require further data and is outside the scope of the present paper.

However the trends taken together do imply that there is no significant contribution to the emission lines from shocks based 


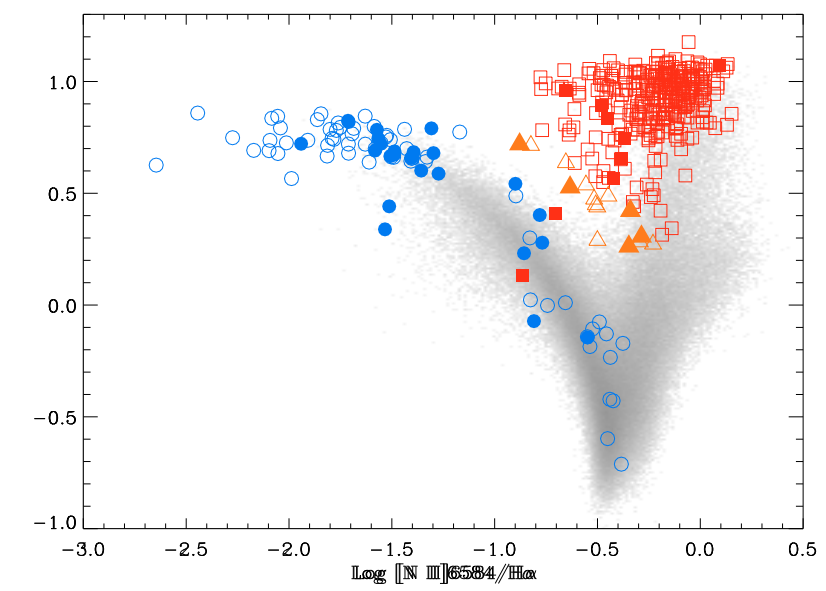

Fig. 12. The distribution of galaxies showing nebular He II $\lambda 4686$ with $S / N>3.5$ in the BPT diagram. The filled symbols mark objects that also show WR signatures in their spectra. The symbols and colours are otherwise as in Fig. 11.

on the models of Dopita \& Sutherland (1995) in good agreement with the results of BPC08 who find that strongly starbursting galaxies in the SDSS do not show any significant contribution to their emission line fluxes from shocks.

\section{The origin of He II nebular emission}

The previous section indicated an increased ionisation parameter in the WR galaxies but equivocal evidence of a harder ionisation field in these sources. It has been suggested in the past that the hard radiation field of Wolf-Rayet stars causes the nebular He II $\lambda 4686$ occasionally seen in H II galaxies (e.g. Schaerer 1996). Guseva et al. (2000) carried out a careful examination of this issue but were not able to show conclusively that the cause of the nebular He II was Wolf-Rayet stars. Here we re-examine the issue, using the small set of SDSS galaxies that show nebular He II $\lambda 4686$ emission. If we focus on those that show He II at $S / N>7(3.5)$ we find a total of 318 (1461) galaxies with nebular He II. Out of these 288 (1392) are at $z>0.01$ and most of these, 269 (1222), appear to by AGN dominated with only 15 (81) being dominated by star-formation. These are plotted on top of the BPT diagram in Fig. 12 where it is clear that the star-forming sources with nebular He II $\lambda 4686$ have low [N II]/H $\alpha$. The solid symbols indicate the location of objects that also show WR features in their spectra.

Figure 13 shows the $\mathrm{He}$ II $\lambda 4686 / \mathrm{H} \beta$ diagram for starforming galaxies in the SDSS DR6. Only galaxies that have He II $\lambda 4686$ detected at $S / N>3.5$ have been included. Note that the $\mathrm{SF}$ galaxies with $\mathrm{He}$ II $/ \mathrm{H} \beta>0.1$ have unreliable $\mathrm{He}$ II and/or $\mathrm{H} \beta$ measurements.

The figure shows a correlation between $\mathrm{O} / \mathrm{H}$ and $\mathrm{He}$ II/ $\mathrm{H} \beta$ such that $\mathrm{He} \mathrm{II} / \mathrm{H} \beta \propto-0.8 \log \mathrm{O} / \mathrm{H}$ over the range $7.6<$ $\log \mathrm{O} / \mathrm{H}<8.0$. This is apparently not due to an increased number of WR stars at low metallicity: the blue points in Fig. 13 show the location of galaxies with detected WR features, and these do not appear to dominate at low metallicity.

We also notice that there appears to be a levelling off at $\log \mathrm{O} / \mathrm{H}<7.6$. At lower metallicity there is a rather broad range in $\mathrm{He} \mathrm{II} / \mathrm{H} \beta$, but no continuation of the trend seen at higher metallicity. We note that this is not likely to be a selection effect because such strong He II lines are easy to detect. The physical

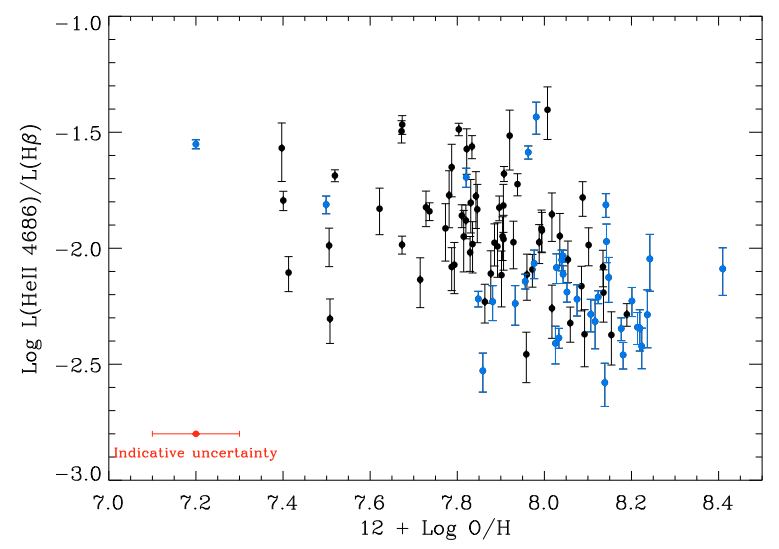

Fig. 13. The $\mathrm{He} \mathrm{II} / \mathrm{H} \beta$ ratio as a function of metallicity for the SDSS DR6. Only galaxies with He II $\lambda 4686$ detected at $S / N>3.5$ and classified as SF galaxies are included. The blue symbols show those galaxies with detected WR features. The uncertainties in the oxygen abundance have been suppressed for clarity but an uncertainty of $0.1 \mathrm{dex}$ is indicated in the lower left.

reasons for this flattening off are unclear and require more modelling of winds and stellar atmospheres in low metallicity stars.

A similar result was reached by Guseva et al. who concluded that in those galaxies not classified as WR galaxies, the WR feature was too weak to detect. Based on these results it is not clear whether this is the right interpretation or whether low density stellar winds at low metallicity lead to a higher flux of He II ionising photons (Smith et al. 2002b; Hadfield \& Crowther 2007). In this case not only WR stars but also regular O stars are expected to contribute to the He II-ionising flux.

The increase in He II-ionising flux, relative to H-Ionising flux could also have a contribution from other sources. Shocks produce nebular He II, but in star forming regions they are mostly caused by colliding winds and as the stellar winds are expected to be weaker at lower metallicities (e.g. Vink \& de Koter 2005) this would not explain the trend seen. Likewise if the increased He II-ionising flux is caused by X-ray binary evolution, one would require an increased binary fraction with reduced metallicity and in absence of any strong evidence of this we do not view this as a likely explanation. This leaves us with the two scenarios that either the main contribution to the He II ionisation is caused by Wolf-Rayet stars, especially WC stars, or that the main contributors are more massive stars such as $\mathrm{O}$ or WN stars.

The key difference between these two scenarios is the timescale. If the dominant source of ionising flux is WC stars, the onset of nebular He II $\lambda 4686$ would be later than in the case of $\mathrm{WN}$ or $\mathrm{O}$ stars being the primary sources.

Figure 14 shows the fraction of galaxies with nebular He II $\lambda 4686$ as a function of $E W(\mathrm{H} \beta)$ in two different abundance regimes. The abundance ranges were chosen to have $50 \mathrm{He}$ II emitters in each and we use $E W(\mathrm{H} \beta)$ as a proxy for age of the burst. However we also indicate the age corresponding to a given $E W(\mathrm{H} \beta)$ assuming that the burst has a duration of $1 \mathrm{Myr}$ on the top $x$-axis. The dashed line shows the fraction of WR galaxies as a function of $E W(\mathrm{H} \beta)$.

From this figure it appears that at low metallicity the WR galaxies, and by implication the WR stars, are not the main cause of nebular He II $\lambda 4686$ emission because the timeevolution is noticeably different. This indicates that regular O stars at low metallicity indeed show a significant emission of photons with $\lambda<228 \AA$ (cf. Smith et al. 2002b; 


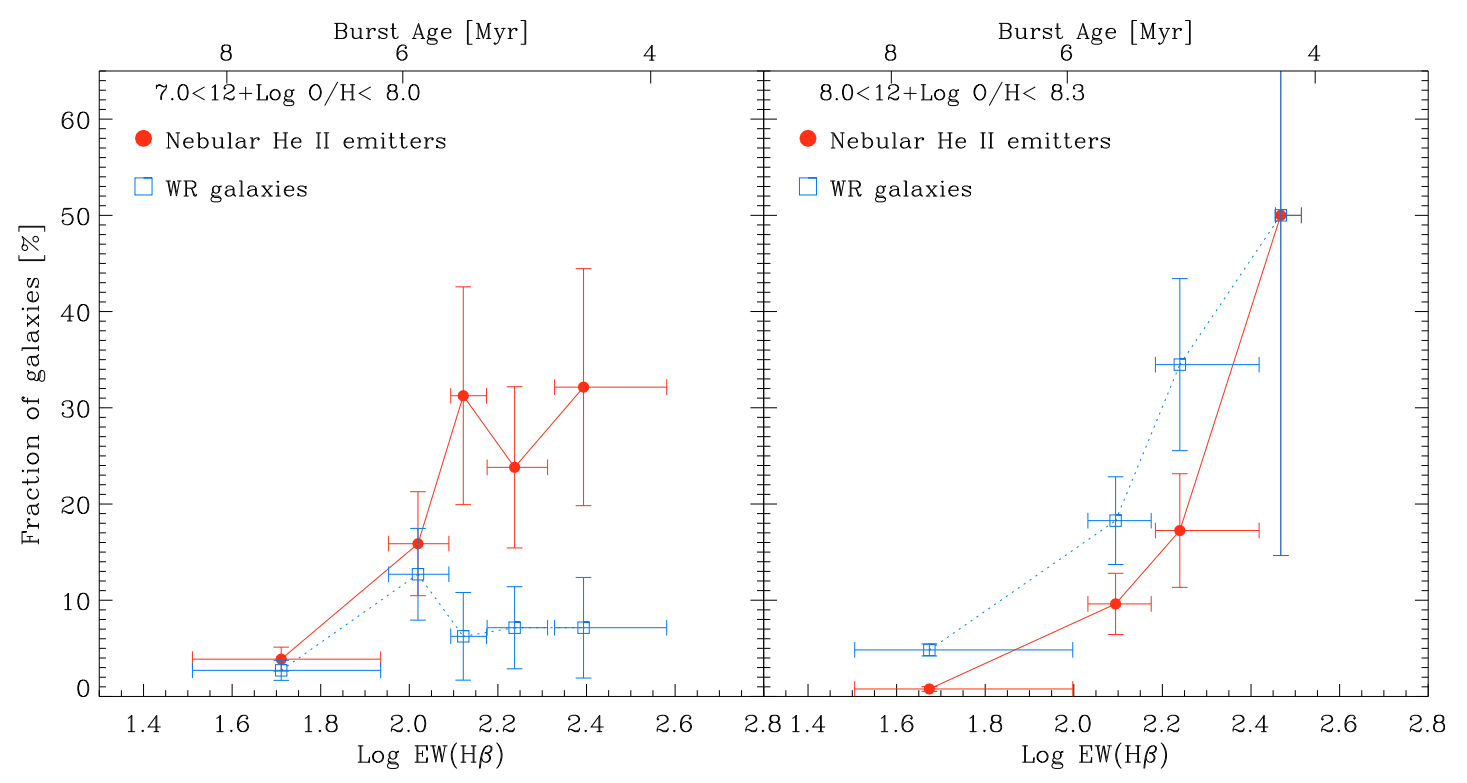

Fig. 14. In the left panel the solid line shows the fraction of star-forming galaxies with $7<12+\log \mathrm{O} / \mathrm{H}<8$ that show nebular He II $\lambda 4686$ in their spectra as a function of $E W(\mathrm{H} \beta)$. The error bars are Poissonian and each bin contains 10 He II $\lambda 4686$ emitters. The upper $x$-axis shows the burst age in Myr corresponding to the $E W(\mathrm{H} \beta)$, see text for details. The dashed line connecting open squares shows how the fraction of WR galaxies in the same abundance range varies with $E W(\mathrm{H} \beta)$. The right panel shows the same for the abundance range $8<12+\log \mathrm{O} / \mathrm{H}<8.3$.

Hadfield \& Crowther 2007). On the other hand at higher metallicity the two show qualitatively similar trends with the WR lines shifted to lower $E W(\mathrm{H} \beta)$. This is consistent with the sources producing the majority of the $<228 \AA$ photons having shorter lifetimes than the WR phase as a whole and that they originate at an early stage in the evolution of a star burst. It is not possible on the basis of these data to make a statement on the relative importance of WN or O stars to the ionisation of He II.

One might worry that the fraction of galaxies harbouring nebular He II emission does not reach $100 \%$, while the argument above would indicate that all sufficiently young star-forming regions should show nebular He II emission. The reason for this apparent discrepancy is the $S / N$ requirement for detecting nebular He II. All the galaxies for which we have detection of nebular He II with $S / N>3$, have a median $S / N$ in their spectra higher than 10 and show a fairly flat distribution in the $S / N$ of their spectra. In contrast, the $S / N$ distribution for spectra with $E W(\mathrm{H} \beta)>30 \AA$ is sharply peaked towards low $S / N$. This does mean that the absolute vertical scale in Fig. 14 is suspect. The appropriate way to deal with this would be to enforce a particular distribution in $S / N$, but the sample is not big enough to do this rigourously. However when simply drawing the comparison sample from a distribution in $S / N$ similar to that of the galaxies showing nebular He II, the fraction of galaxies with nebular He II does tend to $100 \%$ at high $E W(\mathrm{H} \beta)$. A similar conclusion is reached when looking at the $2 \mathrm{D}$ distribution of $S / N$ in the spectra versus $E W(\mathrm{H} \beta)$.

Thus we conclude that our data are consistent with the hypothesis that all systems with $\log E W(\mathrm{H} \beta) \sim 2.5$ show nebular He II as long as the $S / N$ of the spectrum allows its detection. We also conclude that at low metallicity the ionisation of He II is most likely dominated by $\mathrm{O}$ stars, although there could be a contribution of WN stars that we are unable to detect because their features are very weak in the optical. At metallicities higher than $\approx 20 \%$ solar the data are consistent with the major source of ionising radiation being WN stars but a significant contribution from $\mathrm{O}$ stars is possible.

\section{N/O abundance trends and local enrichment}

Since Wolf-Rayet stars have strong winds they should influence their immediate surroundings. However observational evidence of this on large scales has been equivocal. The comprehensive study by Kobulnicky \& Skillman (1996) found no clear difference in the ISM abundance of WR galaxies and other star-burst galaxies. However a number of studies have found spatial variations in the N/O ratio in NGC 5253 (Kobulnicky et al. 1997; López-Sánchez et al. 2007). These studies point to the possibility that winds from Wolf-Rayet stars can mix with the ISM on a relatively short time-scale, in contrast to supernova explosions that require $>10^{8}$ years for the eject to cool enough to allow efficient mixing with the ISM.

In Fig. 15 we show the abundance trends for WR galaxies versus that of non-WR galaxies for $\mathrm{O} / \mathrm{H}, \mathrm{N} / \mathrm{H}, \mathrm{Ar} / \mathrm{H}$ and $\mathrm{Ne} / \mathrm{O}$. It is clear that WR galaxies are in general more metal rich at a given $E W(\mathrm{H} \beta)$ and this is likely a reflection of WR stars being more abundant at higher metallicities as shown in Fig. 8 than WR galaxies. We will therefore focus on abundances relative to oxygen in the following, and as Ar and Ne will turn out to follow oxygen approximately we will also focus mainly on the nitrogen abundance.

The solid line in Fig. 16 shows the median difference in N/O for Wolf-Rayet galaxies and similar galaxies showing no sign of WR stars as a function $E W(\mathrm{H} \beta)$ with the shaded region showing the $1 \sigma$ confidence interval on the median at each value of $E W(\mathrm{H} \beta)$. We see a clear excess $\mathrm{N} / \mathrm{O}$ for WR galaxies at $E W(\mathrm{H} \beta)<100 \AA$. Quantitatively we find $\langle\Delta \log (\mathrm{N} / \mathrm{O})\rangle=0.133 \pm 0.035$ for a $3 \sigma$-clipped mean with errors from bootstrapping.

To calculate this figure we selected for each WR galaxy a set of similar galaxies that show no WR features. These similar galaxies where chosen to have oxygen abundance within 0.1 dex of the WR galaxy and $E W(\mathrm{H} \beta)$ to within a factor of 3 that of the WR galaxies. We then calculate the difference in N/O between the WR galaxies and their similar galaxies. In view of the difference in $\mathrm{O} / \mathrm{H}$ for $\mathrm{WR}$ and non-WR galaxies seen in Fig. 15 it 

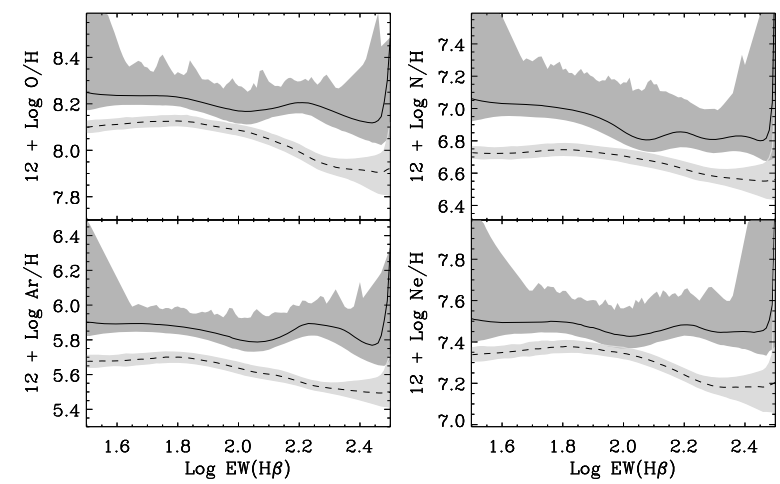

Fig. 15. Top left: the oxygen abundance as a function of $E W(\mathrm{H} \beta)$ for galaxies with WR features in their spectra (solid line and dark shading) and for non-WR galaxies (dashed line and light shading). The lines show the median and the shading the $68 \%$ confidence limit on the median (see text for details of the calculation). The top-right panel shows the same for $12+\log \mathrm{N} / \mathrm{H}$, the bottom left the same for $\mathrm{Ar} / \mathrm{H}$ and the bottom right the same for $\mathrm{Ne} / \mathrm{H}$.

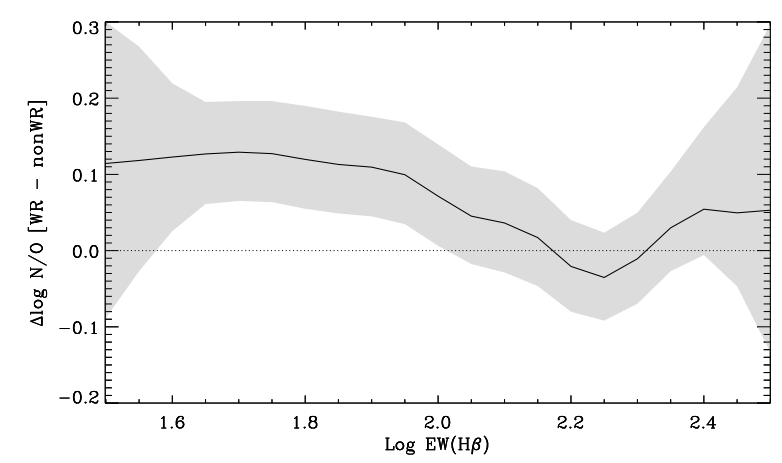

Fig. 16. The difference in N/O between Wolf-Rayet galaxies and similar galaxies showing no sign of Wolf-Rayet stars as a function of $E W(\mathrm{H} \beta)$. The solid line shows the median trend and the the shaded area indicates the $68 \%$ confidence limit on the median (see text for details of the calculation). The dotted line indicates the zero level.

is clear that there will be a difference in N/O between the two classes just due to secondary enrichment of nitrogen if we had not limited our comparison to similar galaxies.

We include all galaxies that are classified as star-forming, have $S / N>5$ in [O III] $\lambda 4363$ and are not duplicate observations of the same region and limit ourselves to spectra for which we can calculate abundances using the $T_{\mathrm{e}}$ method. To estimate confidence limits we bootstrap this procedure 9999 times and for each iteration we draw a new realisation of the relevant observables using their estimated uncertainties, although for $E W(\mathrm{H} \beta)$ we use a flat uncertainty of $2 \AA$, as the error on high $E W(\mathrm{H} \beta)$ lines is dominated by continuum subtraction uncertainties. We emphasise that since the same algorithm is used to calculate abundances regardless of whether a spectrum shows WR features or not, the result is robust to our abundance calculations.

The immediate result from Fig. 16 is that at $E W(\mathrm{H} \beta)>100$, the non-WR and WR galaxies appear to show nearly identical values for $\mathrm{N} / \mathrm{O}$. This is consistent with these being very young bursts where the WR stars have not yet had a chance to enrich the surrounding ISM to a noticeable degree. However at lower $E W(\mathrm{H} \beta)$ there is clear and significant difference between the WR galaxies and the non-WR galaxies in the sense that the former has a higher $\mathrm{N} / \mathrm{O}$ at the same $E W(\mathrm{H} \beta)$. This shows the results for the median trends and it is perhaps even more striking that for $\log \mathrm{N} / \mathrm{O}>-1.3$ and $1.6<\log E W(\mathrm{H} \beta)<2.4$ approximately $25 \%$ of the spectra show WR features, whereas for $\log \mathrm{N} / \mathrm{O}<-1.6$ in the same range in $E W(\mathrm{H} \beta)$, only $4 \%$ of the galaxies show WR features. This is a striking difference and clearly associates an increased N/O with the WR phenomenon.

There are at least two possible explanations for this. The most immediate is that we are seeing an effect of WR winds on the surrounding ISM. This process was already suggested by Pagel et al. (1986) and appears to be the most likely reason for the trends seen.

The second possibility is that this might be a selection effect. WN stars are expected to show CNO in equilibrium proportions in their atmosphere, and as pointed out by Crowther (2000) this leads to the typical WN star to have an earlier type, or in other words to have higher ionisation $\mathrm{N}$ lines in their spectra. The practical consequence for us is that it might be more difficult to classify these stars because the N III $\lambda 4640$ line will become weak. In this case we would be biased against detecting WR features in systems with lower $\mathrm{N}$ abundance. Again this ought to lead to an offset between the WR and non-WR spectra that is constant with $E W(\mathrm{H} \beta)$ in disagreement with what is seen in Fig. 16.

\subsection{Wind pollution of the ISM}

In the preceding we emphasised the role of nitrogen. This is in part because this is expected to be the most sensitive to stellar wind ejecta, but we have also carried out a similar study for $\mathrm{Ne} / \mathrm{O}$ and $\mathrm{Ar} / \mathrm{O}$ and we find no comparable increase in these ratios for the WR galaxies, although the uncertainties are larger. This is expected if the nitrogen enhancement is due to a wind but would not be expected if the enhancement was due to enrichment from SNe. As pointed already by Pagel et al. $(1986,1992)$ and emphasised by Kobulnicky et al. (1997) one should also expect an elevated $\mathrm{He} / \mathrm{H}$ if the observed region has been polluted by the ejecta of WR stars. We are not able to confirm this - the two samples have identical $\mathrm{He} / \mathrm{H}$ to within the uncertainties. Is this consistent with the idea that the increased $\mathrm{N}$ abundance is due to the WR wind?

To answer this we make the simple assumption that the element abundances found in ring nebulae around Galactic WR stars measured by Esteban et al. (1992) are reasonably representative of the heavy element yields of WR winds. These observations indicate that the expected average increase in $\log \mathrm{He} / \mathrm{H}$ in WR ejecta is 0.18 dex whereas the increase in $\log \mathrm{N} / \mathrm{O}$ is $\sim 0.85$ dex so one must expect a higher sensitivity to changes in $\mathrm{N} / \mathrm{O}$ than in $\mathrm{He} / \mathrm{H}$ and our lack of difference in $\mathrm{He} / \mathrm{H}$ between the two samples is fully consistent with the expectations.

It is also important to estimate whether the observed increase in N/O is expected with what one expects based on the output of WR winds. Starting by estimating the mass of ionised hydrogen from the luminosity of $\mathrm{H} \beta, L_{\mathrm{H} \beta}$, through (e.g. Dopita \& Sutherland 2003):

$$
M_{\text {ionised }}=\frac{m_{\mathrm{H}} * L_{\mathrm{H} \beta}}{1.235 \times 10^{-25} T_{4}^{-0.86} n_{\mathrm{e}}},
$$

where $m_{\mathrm{H}}$ is the mass of the hydrogen atom, $T_{4}$ the electron temperature in units of $10000 \mathrm{~K}$ and $n_{\mathrm{e}}$ the electron density.

To quantify the nitrogen enrichment we used the sample of similar galaxies discussed above to calculate

$\Delta\left(\frac{\mathrm{N}}{\mathrm{H}}\right)=\left(\frac{\mathrm{N}}{\mathrm{H}}\right)_{\mathrm{WR}}-\left(\frac{\mathrm{N}}{\mathrm{H}}\right)_{\mathrm{ref}}$, 


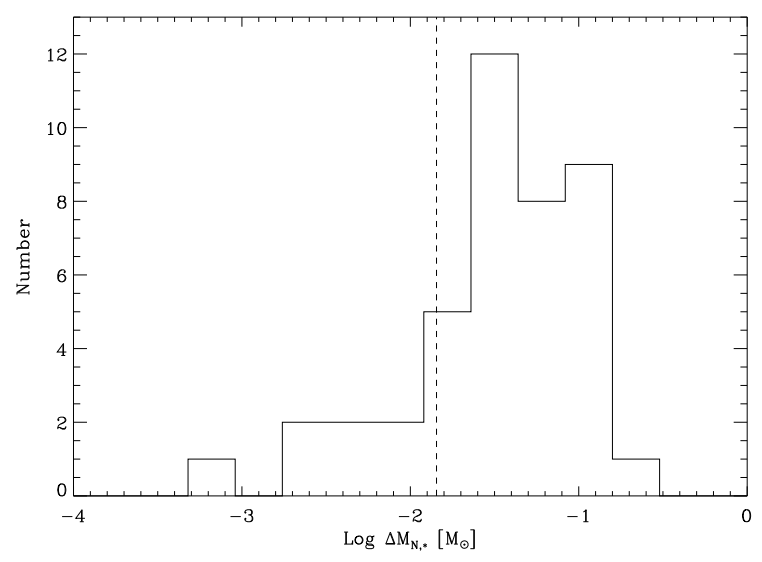

Fig. 17. The distribution of mass of nitrogen ejected in each system per star, $\Delta M_{N, *}$, see text for details. The dashed vertical line shows the mass of nitrogen expected from a mass loss of $10^{-5} M_{\odot} / \mathrm{yr}$ over $2 \mathrm{Myr}$ with the abundance pattern consistent with Galactic WR ring nebulae.

where $(\mathrm{N} / \mathrm{H})_{\text {ref }}$ is the average nitrogen abundance in the galaxies similar to the WR galaxy. This way we can calculate the mass of nitrogen required to explain the offsets

$\Delta M_{\mathrm{N}}=\frac{m_{\mathrm{N}}}{m_{\mathrm{H}}} M_{\text {ionised }} \Delta\left(\frac{\mathrm{N}}{\mathrm{H}}\right)$.

This gives the mass of nitrogen for each system. To estimate the mass of nitrogen ejected per star we need an estimate of the number of WR stars that have contributed to the enrichment process. This is non-trivial to estimate so we make the simple assumption that the present Wolf-Rayet population is a good estimate of the stars involved in the enrichment process. In effect we assume that the WR stars observed are the result of the enrichment process. Given the approximate nature of these estimates we approximate the number of WR stars by dividing the blue bump luminosity, $L_{\mathrm{BB}}$, by a fiducial luminosity, $L_{\mathrm{ref}}$, taken to be $5 \times$ $10^{35} \mathrm{erg} / \mathrm{s}$, giving $N_{\mathrm{WR}}^{L}=L_{\mathrm{BB}} / L_{\mathrm{ref}}$.

We would like to compare these quantities to the nitrogen ejected in Wolf-Rayet ring nebulae. In the Milky Way Esteban et al. (1992) found N/O -0.47 (see also Chu et al. 1999). This gives the total mass nitrogen ejected by stellar winds with a chemical composition like that of Galactic WR ring nebulae

$M_{\mathrm{N}}^{\text {ring }}=\left(\frac{m_{\mathrm{N}}}{m_{\mathrm{H}}}\right)\left(\frac{\mathrm{O}}{\mathrm{H}}\right) M_{\text {ejected }}\left(\frac{\mathrm{N}}{\mathrm{O}}\right)_{\text {ring }}$,

where $M_{\text {ejected }}$ is the total mass ejected from a star. As a rough approximation we take this to be $10^{-5} M_{\odot} /$ yr over a period of $2 \mathrm{Myr}$, or equivalently, a total mass loss of $20 M_{\odot}$ per star. We have here ignored the depletion of oxygen found in ring nebulae by Esteban et al. (1992) which is less obvious in the resolved studies of NGC 5253.

We then approximate the amount of $\mathrm{N}$ ejected per star, $\Delta M_{\mathrm{N}, *}$, by $\Delta M_{\mathrm{N}}$ divided by $N_{\mathrm{WR}}^{L}$. We plot this in Fig. 17 and the median $M_{\mathrm{N}}^{\text {ring }}$ is indicated by the dashed line. Given the rough estimates the agreement is encouraging. Indeed, our calculations assumed that only the currently visible WR population has contributed to the enrichment process, but there might indeed have been additional sources in the past which would bring the two estimates into better agreement. While there could also be a contribution from OB stars, given their observed elevated surfaced abundance (e.g. Hunter et al. 2008, 2007), it is not clear that this would cause a systematic offset at low $E W(\mathrm{H} \beta)$.

\section{Modelling Wolf-Rayet features}

In the preceding we have carried out an empirical study of our sample and the trends seen are in qualitative agreement with the trends seen when studying individual Wolf-Rayet stars in nearby galaxies. However it is well-known (e.g. Legrand et al. 1997; Guseva et al. 2000; Fernandes et al. 2004; Zhang et al. 2007) that there is a significant discrepancy between the predictions of models for Wolf-Rayet stars and the observations at low metallicity. This has led a number of authors to explore models with modified IMFs, the most recent study being that of Zhang et al. (2007). Given the scant observational evidence in favour of a variable IMF we feel it is more appropriate at this point to assess the weaknesses and strengths of models for WR features before appealing to a change in the IMF.

To gain a more thorough understanding of the properties of the galaxies in our sample we need to model the luminosity of the Wolf-Rayet features using stellar evolutionary models. We base ourselves on the methodology of Schaerer \& Vacca (1998, SV98), who presented a model for the spectral evolution of WR stars including their emission lines. The basic framework of this model has since been included in the Starburst 99 stellar population synthesis code (Leitherer et al. 1999) with refined stellar spectra from Smith et al. (2002b). We have adopted this code with the modifications discussed below, to produce instantaneous burst models for the evolution of Wolf-Rayet features.

When comparing measured WR features to models it is common to use very simple star formation histories, either singleburst or constant star formation (e.g. SV98; Guseva et al. 2000; Fernandes et al. 2004; Meynet \& Maeder 2005; Zhang et al. 2007). While appealing because of its simplicity there are two main problems with the approach. The first is that it is commonly assumed that $E W(\mathrm{H} \beta)$ can be used as an age indicator for the bursts in question. While it is often acknowledged that this is an uncertain approximation it is then adopted for comparison to models (e.g. Zhang et al. 2007). However for large samples of WR galaxies spanning a wide range in metallicity and size this might lead to strong biases.

The second problem is that while an assumption of an instantaneous burst is a reasonable approximation for the analysis of H II-regions, it is less ideal for the analysis of our data since we probe larger regions of the galaxy where the star formation history is likely to be more complex. Thus we have combined these predictions from Starburst 99 with the Bruzual \& Charlot (2003, BC03) models to generate predictions for the different Wolf-Rayet emission lines for a wide range of star formation histories.

SV98 based their models on the best empirical compilations of Wolf-Rayet line fluxes available at the time, and while this has stood the test of time quite well, a number of refinements have been published recently. We have updated the line fluxes used by SV98 with the results of the study by Crowther \& Hadfield (2006) who found that lower metallicity systems show a lower He II luminosity and who provide line luminosities for a more uniformly analysed sample of WN stars. As discussed in Brinchmann et al. (2008, BPC08), we have adopted their WN class 5 as representative for WNE stars. For WC stars we have adopted the same luminosities in SV98 as reference.

He II lines are also produced by O If stars. At high metallicity these do not contribute greatly to the He II line luminosity, contributing just a few $\%$ around solar metallicity. However at low metallicity the Wolf-Rayet phase is less prominent and coupled with the reduced line luminosity this does mean that contribution to the He II4686 line for O If stars is more important. The 


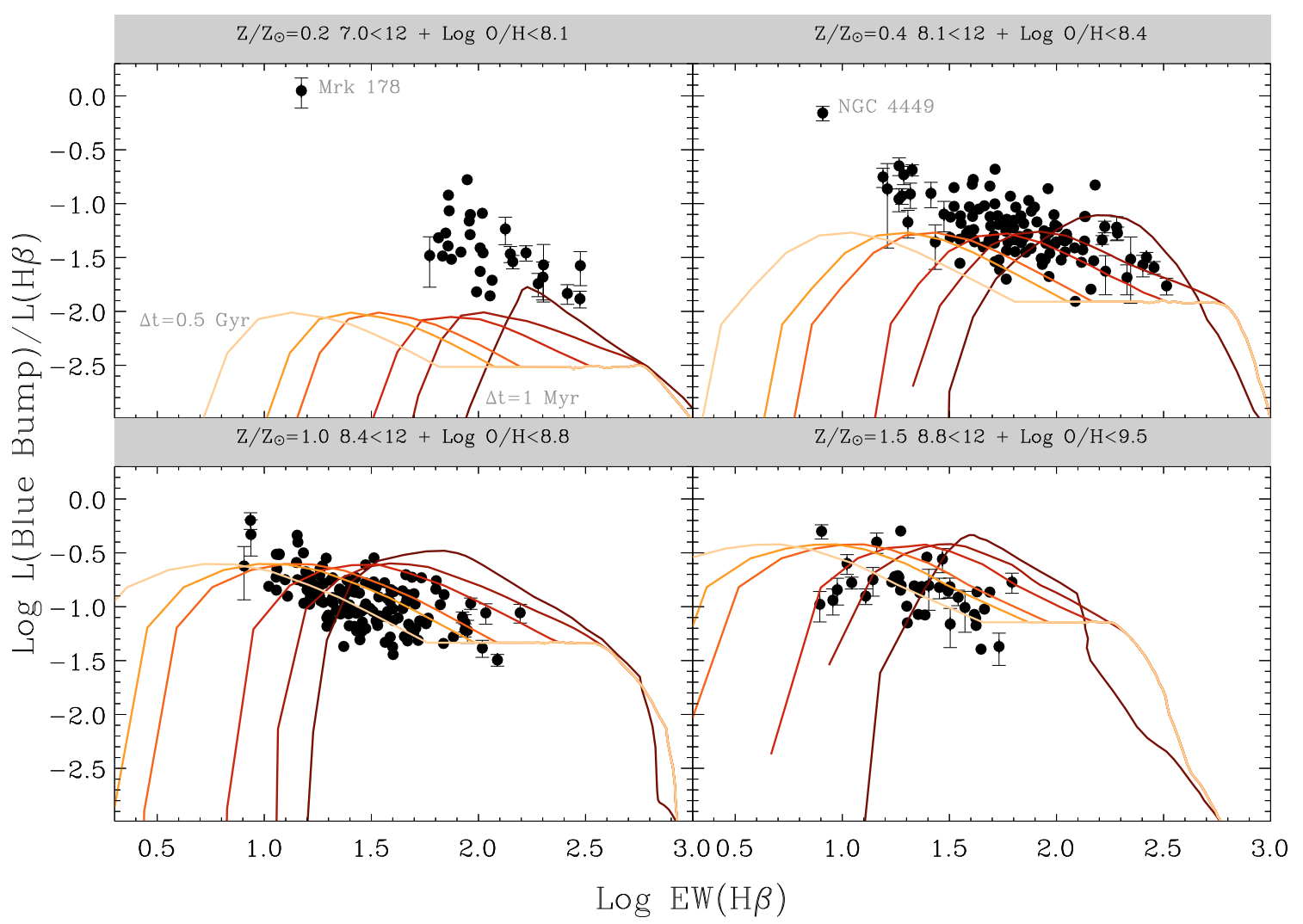

Fig. 18. The blue bump luminosity relative to the $\mathrm{H} \beta$ luminosity as a function of $E W(\mathrm{H} \beta)$ in four different metallicity bins. The range in oxygen abundance used for each bin is indicated in the grey box above each panel. The stellar metallicity adopted for the theoretical tracks is also indicated in this box and are $Z / Z_{\odot}=0.2, Z / Z_{\odot}=0.4, Z=Z_{\odot}$ and $Z / Z_{\odot}=1.5$ respectively. The coloured lines show the tracks of the models described in the text. Moving from left to right they are for burst duration $0.5 \mathrm{Gyr}$ down to $1 \mathrm{Myr}$ in steps of a factor of 2 .

luminosity of He II from these stars was provided by P. Crowther (2007, priv. comm.) and is given in Table 1 in BPC08.

However even with these improvements in modelling, the fact remains that the predictions of the models rely on a number of steps that are not necessarily robust. The assignment of observed Wolf-Rayet properties to stellar tracks is somewhat uncertain and as pointed out by BPC08 there is a significant spread in the observed He II 4686 luminosities even at near constant metallicity and hence predictions are sensitive to the properties of the most extreme stars in any star formation episode.

Furthermore the exact stellar tracks followed by massive stars suffer from uncertainties, both due to rotation (e.g. Meynet $\&$ Maeder 2003, 2005) and to wind loss and binary evolution (e.g Van Bever \& Vanbeveren 2003; Vanbeveren et al. 2007; Eldridge et al. 2008). We focus on single-star models for now and use the tracks of the Geneva High Mass loss tracks of Meynet et al. (1994) since full sets of tracks including rotation are not yet available. However as the minimum masses for the onset of the WR phase are relatively similar in these tracks and those including rotation by Meynet \& Maeder (2005), we do not expect substantial differences, a fact supported by the study of Vázquez et al. (2007).

\subsection{Comparison to models}

The luminosity of the WR features and their strength relative to other spectral properties in our spectra depend on both age, star formation history and metallicity. To simplify comparisons we have grouped our spectra into four metallicity bins according to the oxygen abundance estimate, similar to the bins adopted by
Guseva et al. (2000). We assign galaxies with $12+\log \mathrm{O} / \mathrm{H}<8.1$ to the models with $Z=0.2 Z_{\odot}$. For the most metal poor galaxies this model metallicity is too high, but we will see that even with this conservative model, the discrepancies between models and data are substantial. The $Z=0.4 Z_{\odot}$ models are compared to galaxies with $8.1<12+\log \mathrm{O} / \mathrm{H}<8.4$ and the range $8.4<12+\log \mathrm{O} / \mathrm{H}<8.8$ is compared to solar metallicity tracks, assuming a solar abundance of $\mathrm{O} / \mathrm{H}=8.65$ (Asplund et al. 2004). For galaxies with higher oxygen abundances we compare to the predictions of $Z=1.5 Z_{\odot}$ models which are obtained from the $Z=Z_{\odot}$ and $Z=2.5 Z_{\odot}$ models by linear interpolation in $\log Z$.

Figure 18 shows a comparison of the ratio of the blue bump luminosity to the $\mathrm{H} \beta$ luminosity as a function of the equivalent width of $\mathrm{H} \beta$ (see also Fig. 10). The different panels correspond to the different metallicity bins as indicated above each panel. The uncertainty estimates are shown when there are less than 5 galaxies in bins of $0.1 \mathrm{dex}$ in $\log E W(\mathrm{H} \beta)$.

To illustrate the dependence on star formation history we show tracks for different burst durations, $\Delta t$. The left-most track is for $\Delta t=0.5 \mathrm{Gyr}$ and the right-most track for $\Delta t=1 \mathrm{Myr}$ with the ones in between differing by a factor of 2 in $\Delta t$. A similar effect would be seen when adding an underlying stellar population. $L$ (bluebump) $/ L(\mathrm{H} \beta)$ increases with time until the end of the burst when both lines fade away.

It is obvious that the models do not cover the range of the data in the lowest metallicity bin, and indeed for the most extreme galaxy in the sample, Mrk 178, the discrepancy is close to 2 orders of magnitude. It is however interesting to note that the gradient with respect to $E W(\mathrm{H} \beta)$ is fairly similar in the model 


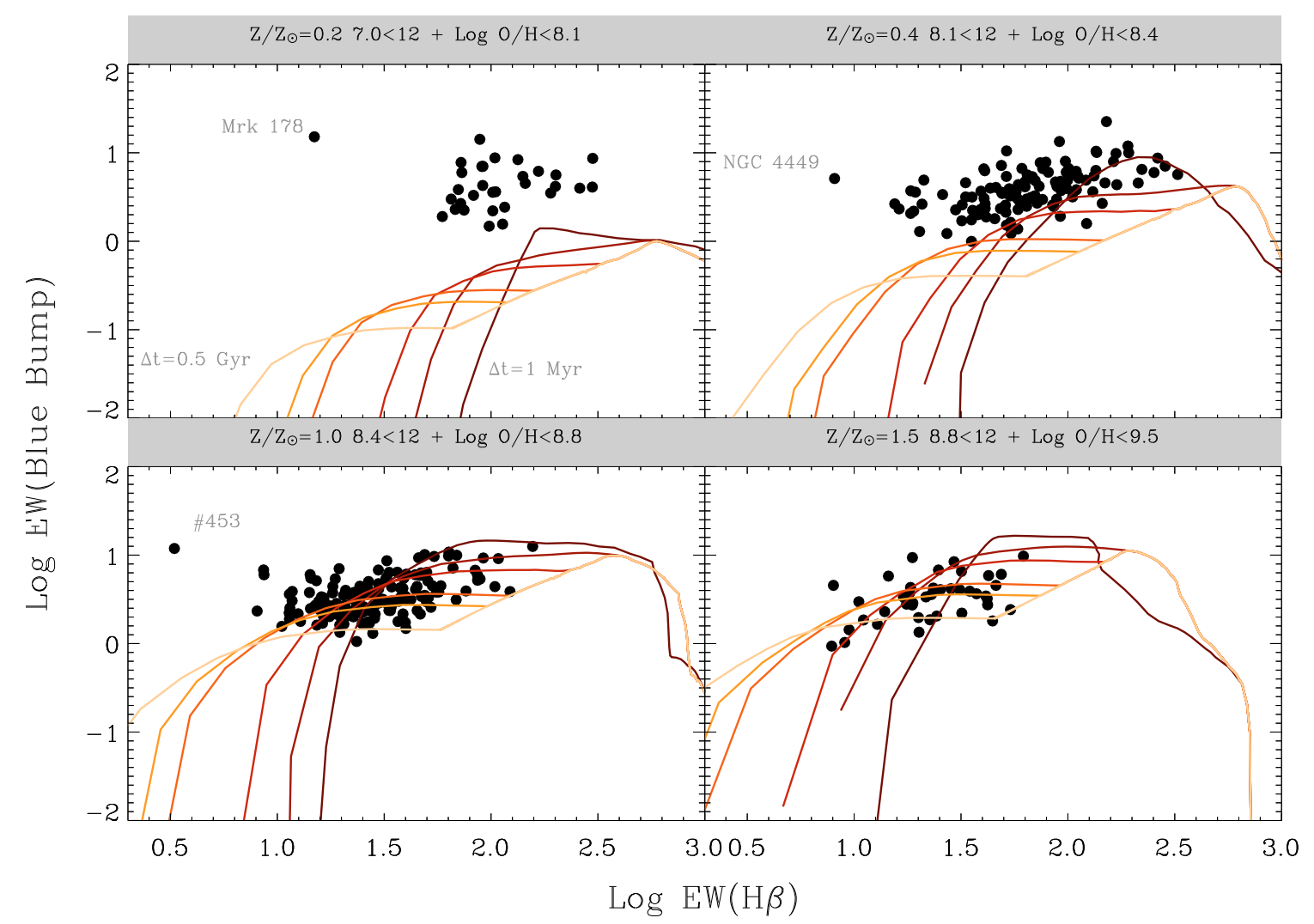

Fig. 19. The equivalent width of the blue bump as a function of the equivalent width of the $\mathrm{H} \beta$ line in different metallicity ranges. The model tracks are as in Fig. 18.

and in the data; this could be interpreted to say that the timescale of the WR evolution is approximately correct but that the absolute number of WR stars is strongly underpredicted.

The $40 \%$ solar models are in better agreement with the data, especially when it is recalled that the curves can be translated to the left by adding an underlying older population or if there is different attenuation of the continuum and lines (e.g. Calzetti 1997). It is however clear that even with this caveat the current models do underpredict the strength of the WR features to a certain degree.

For solar and super-solar metallicities the agreement is good between models and data as one might expect since this is where the observational data on Wolf-Rayet stars is best and where the evolutionary models for stars have been better tested. Indeed it is clear that where the models are well-constrained by observations in the LMC and Milky Way the agreement between models and data is satisfactory but for lower metallicity systems it is increasingly poor.

A similar conclusion can be reached by looking at the equivalent width of the blue bump relative to $E W(\mathrm{H} \beta)$ as shown in Fig. 19. This figure uses the same metallicity bins and model tracks. However in contrast to the preceding figure, an addition of an underlying population shifts the model tracks down and to the left. Thus it is hard to reconcile even the $40 \%$ solar metallicity models with the data. Otherwise the agreement between data and models is as seen before.

\subsection{The origin of the discrepancies}

The mismatches between models and data have been known for some time now (e.g. Legrand et al. 1997; Guseva et al. 2000; Crowther \& Hadfield 2006) although they have usually not been made as explicit because previous studies have formulated the mismatch using derived quantities such as the number of WR stars, $N(\mathrm{WR})$. However in view of what we have seen above we feel it is inappropriate to attempt to derive the number of WR stars using this kind of approach for the full sample, so we have focused on a comparison in the observed frame.

As mentioned above, previous studies have often attempted to explain the discrepancy between observations and models by appealing to a change in the IMF. While we cannot rule this out with the current data we note that there is a lack of independent observational support for a significant change in the IMF with metallicity so we have opted here to fix the IMF.

This leaves us with a significant, up to an order of magnitude, discrepancy between models and data at low metallicity. This is of similar magnitude to that identified by Legrand et al. (1997) in their study of I Zw 18 and recently confirmed by Crowther \& Hadfield (2006). Since cosmological GRBs appear to originate in low metallicity hosts (e.g. Prochaska et al. 2007) and potentially from Wolf-Rayet progenitors, resolving this issue is of major importance not only from the point of view of WolfRayet star formation but also to understand the possible progenitor population for GRBs.

We emphasise here that the problem is in reproducing the amount of Wolf-Rayet stars relative to OB stars (Fig. 18) and relative to the full underlying stellar population (Fig. 19). Since we make the assumption that the IMF is constant there are three possible sources for the discrepancy (see also Crowther 2007, for a similar discussion).

Firstly, it could be that the maximum line luminosities of the WR lines starts to increase again at the lowest metallicities. Since this requires a reversal of the trend towards weaker lines 
at lower metallicities (Crowther \& Hadfield 2006) this appears very unlikely and indeed probably unphysical.

The second possibility is that the life-time of the Wolf-Rayet phase is longer at low metallicity than in the models used here. This appears to be the case for models including rotation as seen in the work of Vázquez et al. (2007). However it is not nearly sufficient to bridge the gap between models and data at low metallicity.

The final possibility is related to the previous, namely to produce more WR stars than currently favoured by the models. As discussed by Crowther (2007) this can either come from changes in the stellar tracks when rotation is introduced or when binarity is included in the evolutionary codes. It is well known that including rotation in the stellar evolution lowers the minimum mass required to form a Wolf-Rayet star (Meynet \& Maeder 2005), but the differences to the Geneva high mass-loss tracks used here are small at metallicities of $40 \%$ solar and above.

The effect of binaries on the evolution of massive stars has been studied quite extensively although the number of relatively poorly known parameters have meant that it is rarely included in population synthesis codes (see Han et al. 2007; Van Bever \& Vanbeveren 2003, for two exceptions). However this is another promising source for an increased WR population although initial results of surveys for binarity among the WR population in the Magellanic clouds did not find an increased binarity at low metallicity (Foellmi et al. 2003b,a).

Quantitatively the requirement from Fig. 18 is that to achieve a match, except for NGC 4449 , for the $Z=0.4 Z_{\odot}$ case we need to increase the predicted blue bump luminosity by a factor of 2 . This is in fact in good agreement with the increase in WR/O ratio for constant star formation rate, between the tracks we use here and the Geneva tracks with rotation used by Vázquez et al. (2007, their Fig. 7). Thus we do not consider the discrepancy between models and data for the $40 \%$ solar case to be of much concern.

However the situation is rather different for the lowest metallicity. We would need to scale the predictions for the luminosity of the blue bump at $Z=0.2 Z_{\odot}$ by a factor of $7-10$ to match the data, with the exception Mrk 178. This is considerably more than the difference due to rotation shown by Vázquez et al. (2007), although newer versions of their models may reduce this deficiency (Meynet et al. 2008). This might indicate that a binary channel for producing Wolf-Rayet stars is important at very low metallicity as suggested by Legrand et al. (1997). This was investigated by Van Bever \& Vanbeveren (2003) who showed that the inclusion of binaries led to a prolonged WR phase. More recently Eldridge et al. (2008) have shown explicitly how this leads to a increase in $N(\mathrm{WR}) / N(\mathrm{O})$ relative to single star models. At low metallicity this becomes very important because single star models are less efficient at producing Wolf-Rayet stars.

In absence of complete evolutionary tracks for these recent models it is not possible to compare to the data shown in Figs. 18 and 19 . However we can make a first comparison to the models by calculating $N_{\mathrm{WR}} / N_{\mathrm{O}}$ using the data in Fig. 10 . To do this we need to estimate the average blue bump luminosity per WR star and the average $\mathrm{H} \beta$ luminosity per $\mathrm{O}$ star. We do this by fitting a grid of models to the observed colours and $E W(\mathrm{H} \beta)$ as described in Sect. B. The conversion ratio is well-constrained, although we do caution that there might be systematic uncertainties, in particular we might overestimate the luminosity of WR stars at low luminosity.

We plot the inferred $N_{\mathrm{WR}} / N_{\mathrm{O}}$ in Fig. 20. This is very similar to Fig. 10 as the average luminosities vary only weakly with time. The well-known decline with metallicity (e.g.

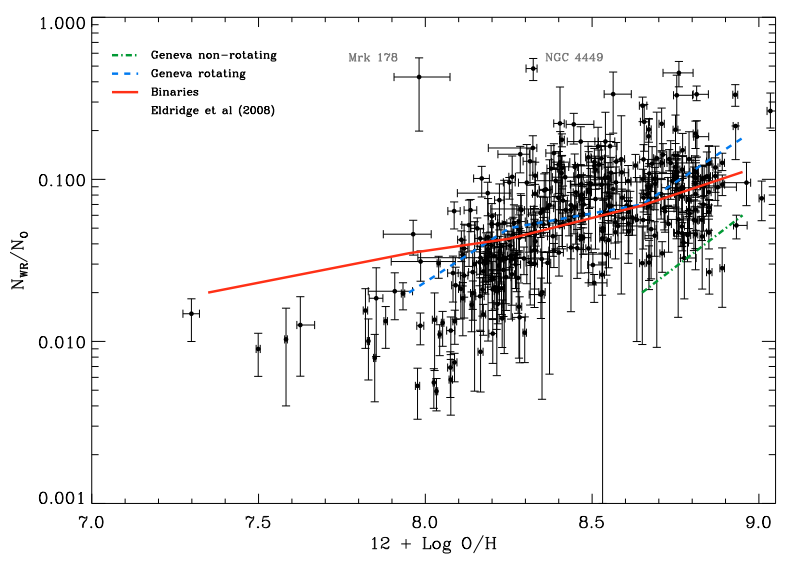

Fig. 20. The ratio of the number of WR stars to that of $O$ stars as a function of oxygen abundance, where we have assumed that the solar oxygen abundance is 8.65 . The solid line shows the predictions of the binary model by Eldridge et al. (2008), the dashed line the Geneva tracks for rotating stars and the dash-dotted line for non-rotating Geneva tracks, both from Meynet \& Maeder (2005).

Crowther 2007) is seen again, but there is clear evidence of a flattening of the relation at low metallicity. This appears to be a robust result as $N_{\mathrm{WR}} / N_{\mathrm{O}}$ might be underestimated at low metallicity if the trend for declining WR luminosity at lower metallicity (Crowther \& Hadfield 2006) continues at metallicities below that of the SMC.

The solid line in the figure shows the values predicted by the models of stellar evolution including massive binaries by Eldridge et al. (2008), assuming a solar oxygen abundance of 8.65. The dashed and dashed-dotted lines show predictions for rotating and non-rotating single stars from Meynet \& Maeder (2005). All predictions are for models with constant star formation and changing the star formation history will move the curves. In view of this and the general model uncertainties, it is reasonable to view agreement within a factor of two as satisfactory.

It is clear that extending the dashed lines to lower metallicity would lead to significant underprediction of WR features and the models without rotation are very strongly ruled out in agreement with various previous studies. The model with binaries on the other hand appears to reproduce the data quite well even at low metallicity. However given that binaries and rotation both give similar predictions at higher metallicity, it is not clear what the relative importance of rotation and binaries will be.

Finally we point out that while the $N(\mathrm{WC}) / N(\mathrm{WN})$ ratio provides a potentially very useful constraint on models for WolfRayet stars (e.g. Schaerer et al. 1999a; Meynet \& Maeder 2005), we have opted to postpone a discussion of this ratio for later work as measurements of red bump fluxes are significantly less secure than that of the blue bump.

In the preceding we did not discuss the highly significant outliers, NGC 4449, our object \#199 and Mrk 178, which we will discuss in some more detail here. Mrk 178 is a starbursting dwarf galaxy in the Canes Venatici group and we adopt a distance of $3.89 \mathrm{Mpc}$ for this object based on the tip of the Red Giant Branch distance from Karachentsev et al. (2003). The oxygen abundance of $12+\log \mathrm{O} / \mathrm{H}=7.73 \pm 0.08$ and the equivalent width of $15.4 \pm 0.4 \AA$ are in excellent agreement with Guseva et al. (2000) who found $7.82 \pm 0.06$ and $15.92 \pm 0.5 \AA$ respectively from an independent observation of the galaxy.

Mrk 178 has one major star forming region to its south where the SDSS spectrum, shown in the top row of Fig. 21, is also 

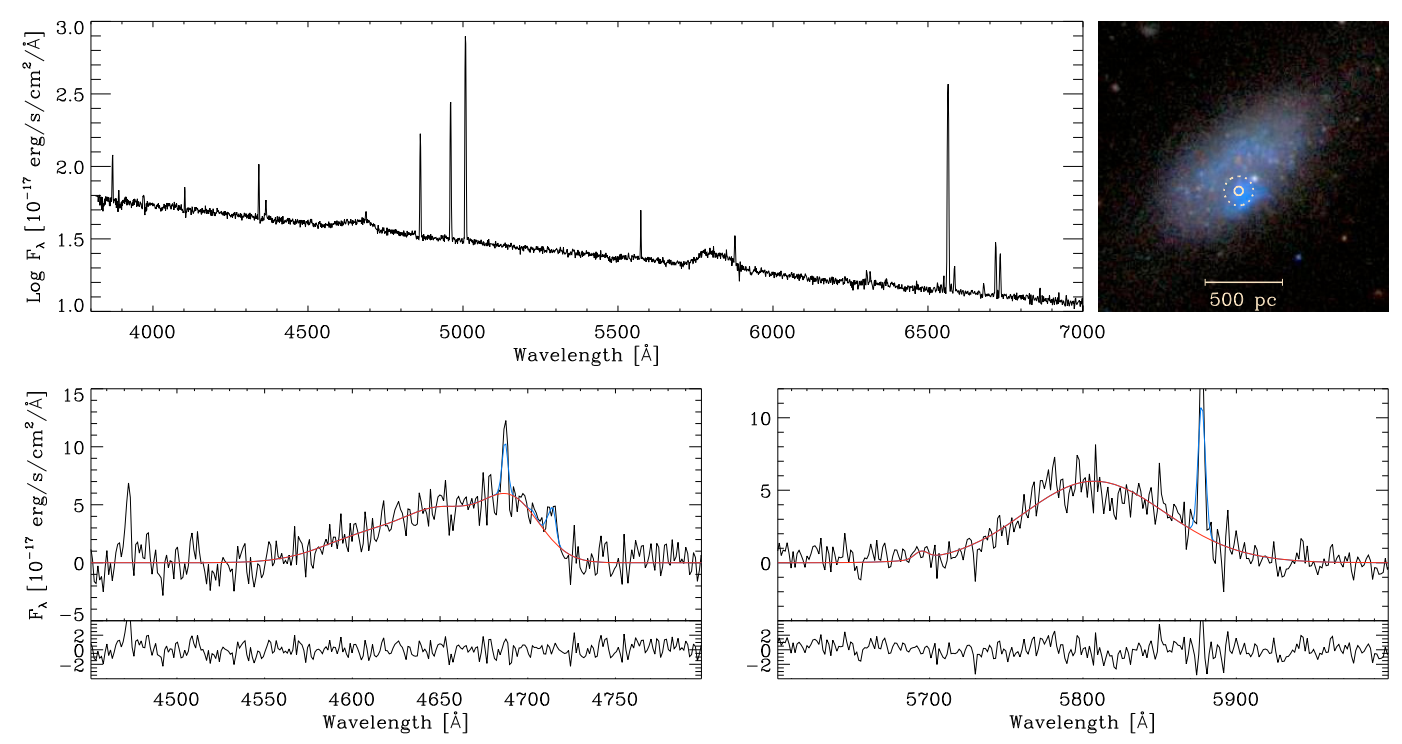

Fig. 21. Top row: on the left, the rest-frame SDSS spectrum of Mrk 178 in units of $\log F_{\lambda}$, the blue and red bump are both very prominent, note also the relatively weak emission lines. On the right a colour image of Mrk 178 from the SDSS with the location of spectroscopic observations indicated by the circles. The outer, dashed, circle is $10^{\prime \prime}$ in diameter while the inner circle shows the size of the SDSS fibre, $3^{\prime \prime}$ in diameter. The bottom row shows the continuum subtracted spectra around the blue bump, on the left, and the red bump on the right, with the residuals after subtracting the fit to the nebular and WR lines indicated in the panel below each as in Fig. 2.

obtained. The prominence of the WR features is obvious and as the bottom row of the figure shows, these are very broad, featureless bumps. The strength of the red bump shows clearly that the main source is WC stars as pointed out by Guseva et al. as well. The immediate impression from Fig. 21 as compared to Fig. 4 is the prominence of the WR features relative to the nebular lines. Indeed, $\log L($ BlueBump $) / L(\mathrm{H} \beta) \approx 0.05$ would convert into a $\log N(\mathrm{WR}) / N(\mathrm{O}) \sim 0.5$, much higher than expected from any model.

However, the luminosity of the blue \& red bumps is only $\sim 10^{37} \mathrm{erg} / \mathrm{s}$, so we expect only a few Wolf-Rayet stars to be sampled. Thus the cause of the offset is almost certainly just that the SDSS fibre, which projects to a size of $56 \mathrm{pc}$, by chance samples a region that is overabundant in Wolf-Rayet stars. This appears also to be the case for NGC 4449, and in this case we also have several spectra additional of star forming regions in NGC 4449, none of which show an offset in the relationships plotted above.

\section{Summary}

We have presented here a study of galaxies with Wolf-Rayet features in their spectra for a carefully selected sample spanning an unprecedented wide range of physical properties. It more than doubles the number of known Wolf-Rayet galaxies and has a well understood selection function. This has allowed us to carry out a number of empirical studies of the abundance of WR stars with metallicity and evolutionary state.

We have shown that by fitting Wolf-Rayet features carefully we can recover the distribution of line widths in Wolf-Rayet stars and we have argued that this shows that we can accurately recover the flux of the Wolf-Rayet features. This has resulted in a sample of WR galaxies with a completeness limit of $E W($ Bluebump $) \approx 1 \AA$ and $E W(\mathrm{H} \beta)>2 \AA$.

We find that the abundance of Wolf-Rayet stars is a strong function both of the oxygen abundance of the galaxy as well as of the star formation intensity as measured by the equivalent width of $\mathrm{H} \beta$. Intriguingly we find that above $E W(\mathrm{H} \beta) \sim 200 \AA$ the fraction of galaxies showing signs of WR stars appear to start to decline. While the $E W(\mathrm{H} \beta)$ is a questionable age indicator for the spectra in our sample, this does appear to be consistent with current theoretical predictions for the onset of Wolf-Rayet formation at 1-2 Myr.

We also find that galaxies that show Wolf-Rayet features in their spectra have a nitrogen abundance that is $\approx 0.1$ dex higher than systems that do not show Wolf-Rayet features. We have argued that this appears to be the result of pollution of the ISM from Wolf-Rayet winds. The observed increases in N/O are consistent with the result of typical Wolf-Rayet winds releasing $\mathrm{N}$ into the ISM over a period of a few Myr. The present study is limited by the number of galaxies with high quality nitrogen abundance measurements and it would be very interesting to extend this study to galaxies with higher oxygen abundance and less intense star formation and to empirically determine the region of influence of the WR winds.

Finally we have also examined whether Wolf-Rayet stars are responsible for the ionisation of He II causing the nebular He II $\lambda 4686$. We were able to show that the time-scales for the WR phase and the nebular He II are different at low metallicity and that the dominant contribution of the ionisation of He II here is likely to be massive $\mathrm{O}$ stars. By inference, the winds of low metallicity massive $\mathrm{O}$ stars must be weaker than high metallicity equivalents to allow for the escape of $\lambda<228 \AA$ photons. At higher metallicity we find that it is very likely that WN stars are contributing significantly to the production of He II ionising radiation. While these are model-independent inferences it is clear that it would be very valuable to compare these results with detailed models of high mass stars at low metallicity to understand the evolution of wind strengths with metallicity.

Acknowledgements. We would like to thank Stéphane Charlot, G. Comte, Thierry Contini, Paul Crowther, Tim Heckman, Max Pettini, D. Rosa-Gonzalez, E. and R. Terlevich, D. Schaerer, G. Meynet and J. Eldridge for fruitful discussions and help on various topics in this paper. In particular we gratefully acknowledge feedback on an earlier version of the paper from G. Meynet, D. Schaerer and J. Eldridge. We warmly acknowledge assistance from and discussions with Gilles Missonnier and thank G. Bruzual and S. Charlot for providing an updated version of their models prior to publication. J.B. acknowledges the receipt of FCT grant SFRH/BPD/14398/2003 and support from FCT 
grant PTDC/CTE-AST/66147/2006. The project has a web page at http:// www.strw. leidenuniv.nl/jarle/WRinSDSS/.Funding for the Sloan Digital Sky Survey (SDSS) and SDSS-II has been provided by the Alfred P. Sloan Foundation, the Participating Institutions, the National Science Foundation, the US Department of Energy, the National Aeronautics and Space Administration, the Japanese Monbukagakusho, and the Max Planck Society, and the Higher Education Funding Council for England. The SDSS Web site is http://www . sdss.org/. The SDSS is managed by the Astrophysical Research Consortium (ARC) for the Participating Institutions. The Participating Institutions are the American Museum of Natural History, Astrophysical Institute Potsdam, University of Basel, University of Cambridge, Case Western Reserve University, The University of Chicago, Drexel University, Fermilab, the Institute for Advanced Study, the Japan Participation Group, The Johns Hopkins University, the Joint Institute for Nuclear Astrophysics, the Kavli Institute for Particle Astrophysics and Cosmology, the Korean Scientist Group, the Chinese Academy of Sciences (LAMOST), Los Alamos National Laboratory, the Max-PlanckInstitute for Astronomy (MPIA), the Max-Planck-Institute for Astrophysics (MPA), New Mexico State University, Ohio State University, University of Pittsburgh, University of Portsmouth, Princeton University, the United States Naval Observatory, and the University of Washington. This research has made use of the NASA/IPAC Extragalactic Database (NED) which is operated by the Jet Propulsion Laboratory, California Institute of Technology, under contract with the National Aeronautics and Space Administration. The research has also made extensive use of the VizieR catalogue access tool, CDS, Strasbourg, France as well as NASA's Astrophysics Data System Bibliographic Services. The analysis has also made use of the Perl Data Language (PDL, http://pdl. perl .org), the Interactive Data Language (IDL) and the R project for Statistical Computing (http://www.r-project.org).

\section{Appendix A: Pipeline processing of the spectra}

The spectra of the DR6 have been re-analysed in a two-step process. First, all the spectra were re-analysed using the pipeline outlined by Tremonti et al. (2004, hereafter T04) and also discussed in Brinchmann et al. (2004, hereafter B04) and summarised below. This provides good first estimates of continuum features and emission lines in the SDSS spectra.

However, because spectra showing Wolf-Rayet features often originate in galaxies with particularly elevated star formation activity, the spectra of interest to us are more complex than the vast majority of the SDSS spectra and we have therefore reprocessed the spectra using a new pipeline to more accurately estimate emission line fluxes for very strong lines and also to measure many more lines than is normally done, something that would be very inefficient using the standard pipeline. Furthermore the normal pipeline reduction applies a smooth continuum correction to adjust for errors in the spectrophotometric calibration of spectra, but this smooth correction might indeed obliterate any Wolf-Rayet feature so we have modified this procedure somewhat as discussed below.

The pipeline, platefit, developed by T04 proceeds by first calculating a fit to the absorption line spectrum of a galaxy. Subsequently a set of strong emission lines are fit to the absorption line subtracted spectrum. For this latter process an additional smooth component is calculated to adjust for any nonperfect spectrophotometric calibration of the spectrum.

The key feature of the emission line fit is that the forbidden and Balmer lines are grouped in two groups and for each of these groups the line-width and velocity offset of the lines are assumed to be the same for all lines. This enables us to measure weak lines that otherwise would not be accurately constrained.

The disadvantage of the technique is that the multi-parameter fit might occasionally reach a local minimum rather than the global best fit solution. This problem worsens as more lines are included in the fit because the search space for the minimisation problem becomes higher dimensional.

Thus we have developed a second pipeline which takes the width and velocity offset of the forbidden and Balmer lines from the T04 pipelines and carries out fits of a larger set of emission lines. We adopt the Balmer line widths for the Hydrogen and Helium recombination lines and the forbidden line widths for all the forbidden lines. The lines superposed on the broad WolfRayet features are treated separately as discussed further below.

All the lines considered are then fit jointly where line widths are set to the previously determined values from the T04 fit this allows us to simplify the fitting routine and get good convergence for the joint fits. Occasionally the joint fit might not give a perfect fit for very strong lines, so for each line that has $S / N>$ 10 we redo the fit, this time fitting the line (or where appropriate, a blend) with the line position freely determined as well as the continuum level, we also do the fit allowing the line-width to vary freely and choose the statistically best fit given the number of free parameters. This multi-step process gives excellent fits to all the emission lines considered here and can be applied automatically to all DR6 spectra.

\section{Appendix B: Fitting models to colours and $E W(\mathrm{H} \beta)$}

As mentioned in the text, the spectra in our sample do not represent single burst systems. This means that we need to consider more complex star formation histories but because the Wolf-Rayet phases are very short-lasting we also need high time resolution.

To achieve this we calculated combinations of smoothly varying star formation histories with superposed bursts using the single stellar population models discussed in the text. We constructed a grid of models covering the observed space in $u-g$, $g-r, r-i$ and $E W(\mathrm{H} \beta)$. This grid was parametrised by the underlying star formation history, the time at which a burst starts, $t_{\mathrm{B}}$, the duration of the burst $\Delta t$ and the fraction of final mass formed in the burst, $m_{\mathrm{R}}$.

We use three underlying star formation histories (SFH), a top-hat burst of duration $1 \mathrm{Gyr}$, an exponentially declining SFH with time-constant $1 \mathrm{Gyr}$ and an exponentially declining SFH with time-constant 15 Gyr. The latter is near-identical to a constant SFH.

We calculate models for burst durations between $1 \mathrm{Myr}$ and 0.5 Gyr increasing the duration by a factor of 5 between each step, and introduce bursts on top of the underlying SFHs at 1, 2, 4 and 6 Gyr after commencement of star formation. The bursts contribute from $5 \%$ to $90 \%$ of the final mass in steps of $2.5 \%$. All of these models are calculated for the four metallicities available, $Z=0.004,0.008,0.02,0.05$ and we use a time-step of $0.5 \mathrm{Myr}$ at times later than $10 \mathrm{Myr}$ after the commencement of the burst, and follow the evolution at this resolution until 3 Gyr after the burst.

We then compare the observed $u, g, r, i$ fluxes and $E W(\mathrm{H} \beta)$ to the model predictions and assign each model a likelihood given as $P=\exp \left(-\chi^{2} / 2\right)$, similarly to the approach taken by Kauffmann et al. (2003b).

In the text we only require the conversion factor from $L_{\text {Bluebump }} / L_{\mathrm{H} \beta}$ to $N_{\mathrm{WR}} / N_{\mathrm{O}}$, and this quantity is well constrained by the model fits. However it should be kept in mind that this quantity might be subject to significant unknown systematic uncertainties since we rely on the $L_{\text {Bluebump }}$ distribution observed in the local Universe which does not fully cover the parameters of our sample galaxies.

On the other hand, to get reasonable constraints on the age of the burst it is furthermore required to apply a prior, requiring that the model has to contain enough Wolf-Rayet stars to have $E W($ Blue bump) $>1 \AA$. This is sensitive to model assumptions, and age estimates are poorly constrained at $E W(\mathrm{H} \beta)<100 \AA$ 
even with this prior. We have therefore opted not to use these age estimates in the text.

\section{References}

Adelman-McCarthy, J. K., Agüeros, M. A., Allam, S. S., et al. 2008, ApJS, 175, 297

Allen, D. A., Wright, A. E., \& Goss, W. M. 1976, MNRAS, 177, 91

Allen, S. W. 1995, MNRAS, 276, 947

Armandroff, T. E., \& Massey, P. 1985, ApJ, 291, 685

Asari, N. V., Cid Fernandes, R., Stasińska, G., et al. 2007, MNRAS, 381, 263

Asplund, M., Grevesse, N., Sauval, A. J., Allende Prieto, C., \& Kiselman, D. 2004, A\&A, 417, 751

Baldwin, J. A., Phillips, M. M., \& Terlevich, R. 1981, PASP, 93, 5

Binette, L., Wilson, A. S., \& Storchi-Bergmann, T. 1996, A\&A, 312, 365

Blanton, M. R., Lin, H., Lupton, R. H., et al. 2003, AJ, 125, 2276

Blanton, M. R., Lupton, R. H., Schlegel, D. J., et al. 2005, ApJ, 631, 208

Bresolin, F. 2007, ApJ, 656, 186

Brinchmann, J., Charlot, S., White, S. D. M., et al. 2004, MNRAS, 351, 1151

Brinchmann, J., Pettini, M., \& Charlot, S. 2008, MNRAS, 385, 769

Bruzual, G., \& Charlot, S. 2003, MNRAS, 344, 1000

Calzetti, D. 1997, AJ, 113, 162

Charlot, S., \& Fall, S. M. 2000, ApJ, 539, 718

Charlot, S., \& Longhetti, M. 2001, MNRAS, 323, 887

Chu, Y.-H., Weis, K., \& Garnett, D. R. 1999, AJ, 117, 1433

Conti, P. S. 1991, ApJ, 377, 115

Conti, P. S., Garmany, C. D., \& Massey, P. 1989, ApJ, 341, 113

Contini, T., Kunth, D., Mas-Hesse, J., \& Arribas, A. 2001, EAS Pub. Ser. (Les

Ulis, France: EDP Sciences), 1, 163

Copetti, M. V. F., Pastoriza, M. G., \& Dottori, H. A. 1986, A\&A, 156, 111

Crowther, P. A. 2000, A\&A, 356, 191

Crowther, P. A. 2007, ARA\&A, 45, 177

Crowther, P. A., \& Hadfield, L. J. 2006, A\&A, 449, 711

Crowther, P. A., Hadfield, L. J., Schild, H., \& Schmutz, W. 2004, A\&A, 419, L17

de Mello, D. F., Schaerer, D., Heldmann, J., \& Leitherer, C. 1998, ApJ, 507, 199 Dopita, M. A., \& Sutherland, R. S. 1995, ApJ, 455, 468

Dopita, M. A., \& Sutherland, R. S. 2003, Astrophysics of the diffuse universe (Berlin, New York: Springer), Astronomy and astrophysics library

Dopita, M. A., Fischera, J., Sutherland, R. S., et al. 2006, ApJS, 167, 177

Eldridge, J. J., Izzard, R. G., \& Tout, C. A. 2008, MNRAS, 111

Erb, D. K., Shapley, A. E., Pettini, M., et al. 2006, ApJ, 644, 813

Esteban, C., Vilchez, J. M., Smith, L. J., \& Clegg, R. E. S. 1992, A\&A, 259, 629

Fernandes, I. F., de Carvalho, R., Contini, T., \& Gal, R. R. 2004, MNRAS, 355, 728

Foellmi, C., Moffat, A. F. J., \& Guerrero, M. A. 2003a, MNRAS, 338, 360

Foellmi, C., Moffat, A. F. J., \& Guerrero, M. A. 2003b, MNRAS, 338, 1025

Fukugita, M., Ichikawa, T., Gunn, J. E., et al. 1996, AJ, 111

Gómez, P. L., Nichol, R. C., Miller, C. J., et al. 2003, ApJ, 584, 210

Gunn, J. E., Carr, M., Rockosi, C., et al. 1998, AJ, 116, 3040

Gunn, J. E., Siegmund, W. A., Mannery, E. J., et al. 2006, AJ, 131, 2332

Guseva, N. G., Izotov, Y. I., \& Thuan, T. X. 2000, ApJ, 531, 776

Hadfield, L. J., \& Crowther, P. A. 2006, MNRAS, 368, 1822

Hadfield, L. J., \& Crowther, P. A. 2007, MNRAS, 381, 418

Hamann, W.-R., Gräfener, G., \& Liermann, A. 2006, A\&A, 457, 1015

Hammer, F., Flores, H., Schaerer, D., et al. 2006, A\&A, 454, 103

Han, Z., Podsiadlowski, P., \& Lynas-Gray, A. E. 2007, MNRAS, 380, 1098

Heckman, T. M., \& Leitherer, C. 1997, AJ, 114, 69

Ho, L. C., Filippenko, A. V., \& Sargent, W. L. 1995, ApJS, 98, 477

Hogg, D. W., Finkbeiner, D. P., Schlegel, D. J., \& Gunn, J. E. 2001, AJ, 122, 2129

Hunter, I., Dufton, P. L., Smartt, S. J., et al. 2007, A\&A, 466, 277

Hunter, I., Lennon, D. J., Dufton, P. L., et al. 2008, A\&A, 479, 541

Izotov, Y. I., Stasińska, G., Meynet, G., Guseva, N. G., \& Thuan, T. X. 2006,

A\&A, 448, 955

Karachentsev, I. D., Sharina, M. E., Dolphin, A. E., et al. 2003, A\&A, 398, 467

Kauffmann, G., Heckman, T. M., Tremonti, C., et al. 2003a, MNRAS, 346, 1055

Kauffmann, G., Heckman, T. M., White, S. D. M., et al. 2003b, MNRAS, 341, 33

Kauffmann, G., Heckman, T. M., De Lucia, G., et al. 2006, MNRAS, 367, 1394

Kennicutt, R. C., Bresolin, F., French, H., \& Martin, P. 2000, ApJ, 537, 589

Kennicutt, R. C., Bresolin, F., \& Garnett, D. R. 2003, ApJ, 591, 801

Kewley, L. J., \& Dopita, M. A. 2002, ApJS, 142, 35

Kewley, L. J., \& Ellison, S. L. 2008, ApJ, accepted [arXiv: 0801. 1849]
Kewley, L. J., Dopita, M. A., Sutherland, R. S., Heisler, C. A., \& Trevena, J. 2001, ApJ, 556, 121

Kewley, L. J., Groves, B., Kauffmann, G., \& Heckman, T. 2006, MNRAS, 372, 961

Kniazev, A. Y., Pustilnik, S. A., Grebel, E. K., Lee, H., \& Pramskij, A. G. 2004, ApJS, 153, 429

Kobulnicky, H. A., \& Koo, D. C. 2000, ApJ, 545, 712

Kobulnicky, H. A., \& Skillman, E. D. 1996, ApJ, 471, 211

Kobulnicky, H. A., Skillman, E. D., Roy, J.-R., Walsh, J. R., \& Rosa, M. R. 1997, ApJ, 477, 679

Kunth, D., \& Joubert, M. 1985, A\&A, 142, 411

Kunth, D., \& Sargent, W. L. W. 1981, A\&A, 101, L5

Kunth, D., \& Sargent, W. L. W. 1983, ApJ, 273, 81

Kunth, D., Sargent, W. L. W., \& Kowal, C. 1981, A\&AS, 44, 229

Le Borgne, J.-F., Bruzual, G., Pelló, R., et al. 2003, A\&A, 402, 433

Legrand, F., Kunth, D., Roy, J.-R., Mas-Hesse, J. M., \& Walsh, J. R. 1997, A\&A, 326, L17

Leitherer, C., Schaerer, D., Goldader, J. D., et al. 1999, ApJS, 123, 3

Liu, X., Shapley, A. E., Coil, A. L., Brinchmann, J., \& Ma, C.-P. 2008, ApJ, accepted [arXiv:0801.1670L]

López-Sánchez, Á. R., Esteban, C., García-Rojas, J., Peimbert, M., \& Rodríguez, M. 2007, ApJ, 656, 168

Lowenthal, J. D., Hogan, C. J., Green, R. F., et al. 1991, ApJ, 377, L73

Lowenthal, J. D., Koo, D. C., Guzman, R., et al. 1997, ApJ, 481, 673

Lupton, R., et al. 2008, AJ, submitted

Maeder, A., \& Meynet, G. 1994, A\&A, 287, 803

Mas-Hesse, J. M., \& Kunth, D. 1991, A\&AS, 88, 399

Mas-Hesse, J. M., \& Kunth, D. 1999, A\&A, 349, 765

Meynet, G., \& Maeder, A. 2003, A\&A, 404, 975

Meynet, G., \& Maeder, A. 2005, A\&A, 429, 581

Meynet, G., Maeder, A., Schaller, G., Schaerer, D., \& Charbonnel, C. 1994, A\&AS, 103, 97

Meynet, G., Ekstrom, S., Maeder, A., et al. 2008 [arXiv: 0802 .2805]

Nugis, T., \& Lamers, H. J. G. L. M. 2000, A\&A, 360, 227

Osterbrock, D. E. 1989, Astrophysics of gaseous nebulae and active galactic nuclei, Research supported by the University of California, John Simon Guggenheim Memorial Foundation, University of Minnesota, et al. (Mill Valley, CA: University Science Books), 422

Osterbrock, D. E., \& Cohen, R. D. 1982, ApJ, 261, 64

Pagel, B. E. J., Terlevich, R. J., \& Melnick, J. 1986, PASP, 98, 1005

Pagel, B. E. J., Simonson, E. A., Terlevich, R. J., \& Edmunds, M. G. 1992, MNRAS, 255, 325

Pettini, M., \& Pagel, B. E. J. 2004, MNRAS, 348, L59

Pier, J. R., Munn, J. A., Hindsley, R. B., et al. 2003, AJ, 125, 1559

Pilyugin, L. S. 2000, A\&A, 362, 325

Pindao, M., Schaerer, D., González Delgado, R. M., \& Stasińska, G. 2002, A\&A, 394,443

Prochaska, J. X., Chen, H.-W., Dessauges-Zavadsky, M., \& Bloom, J. S. 2007, ApJ, 666, 267

Sánchez-Blázquez, P., Peletier, R. F., Jiménez-Vicente, J., et al. 2006, MNRAS, 371,703

Schaerer, D. 1996, ApJ, 467, L17

Schaerer, D., \& Vacca, W. D. 1998, ApJ, 497, 618

Schaerer, D., Contini, T., \& Kunth, D. 1999a, A\&A, 341, 399

Schaerer, D., Contini, T., \& Pindao, M. 1999b, A\&AS, 136, 35

Schlegel, D. J., Finkbeiner, D. P., \& Davis, M. 1998, ApJ, 500, 525

Shapley, A. E., Steidel, C. C., Pettini, M., \& Adelberger, K. L. 2003, ApJ, 588, 65

Shimasaku, K., Fukugita, M., Doi, M., et al. 2001, AJ, 122, 1238

Smith, L. F., Shara, M. M., \& Moffat, A. F. J. 1996, MNRAS, 281, 163

Smith, J. A., Tucker, D. L., Kent, S., et al. 2002a, AJ, 123, 2121

Smith, L. J., Norris, R. P. F., \& Crowther, P. A. 2002b, MNRAS, 337, 1309

Steidel, C. C., Pettini, M., \& Adelberger, K. L. 2001, ApJ, 546, 665

Steidel, C. C., Adelberger, K. L., Shapley, A. E., et al. 2003, ApJ, 592, 728

Tremonti, C. A., Heckman, T. M., Kauffmann, G., et al. 2004, ApJ, 613, 898

Tucker, D. L., Kent, S., Richmond, M. W., et al. 2006, Astron. Nachr., 327, 821

Van Bever, J., \& Vanbeveren, D. 2003, A\&A, 400, 63

van der Hucht, K. A. 2001, New Astron. Rev., 45, 135

Vanbeveren, D., Van Bever, J., \& Belkus, H. 2007, ApJ, 662, L107

Vázquez, G. A., Leitherer, C., Schaerer, D., Meynet, G., \& Maeder, A. 2007, ApJ, 663, 995

Vink, J. S., \& de Koter, A. 2005, A\&A, 442, 587

Woosley, S. E., \& Bloom, J. S. 2006, ARA\&A, 44, 507

Yin, S. Y., Liang, Y. C., Hammer, F., et al. 2007, A\&A, 462, 535

York, D. G., Adelman, J., Anderson, J. E., Jr., et al. 2000, AJ, 120, 1579

Zhang, W., Kong, X., Li, C., Zhou, H.-Y., \& Cheng, F.-Z. 2007, ApJ, 655, 851 